University of Nebraska - Lincoln

DigitalCommons@University of Nebraska - Lincoln

$4-12-2008$

\title{
Pesticides in Rain in Four Agricultural Watersheds in the United States
}

Jason R. Vogel

U.S. Geological Survey, jason.vogel@ou.edu

Michael S. Majewski

U.S. Geological Survey

Paul D. Capel

U.S. Geological Survey

Follow this and additional works at: https://digitalcommons.unl.edu/usgsstaffpub

Part of the Earth Sciences Commons

Vogel, Jason R.; Majewski, Michael S.; and Capel, Paul D., "Pesticides in Rain in Four Agricultural Watersheds in the United States" (2008). USGS Staff -- Published Research. 3.

https://digitalcommons.unl.edu/usgsstaffpub/3

This Article is brought to you for free and open access by the US Geological Survey at DigitalCommons@University of Nebraska - Lincoln. It has been accepted for inclusion in USGS Staff -- Published Research by an authorized administrator of DigitalCommons@University of Nebraska - Lincoln. 


\title{
Pesticides in Rain in Four Agricultural Watersheds in the United States
}

\author{
Jason R. Vogel, ${ }^{*}$ Michael S. Majewski, and Paul D. Capel UsGS
}

Rainfall samples were collected during the 2003 and 2004 growing seasons at four agricultural locales across the USA in Maryland, Indiana, Nebraska, and California. The samples were analyzed for 21 insecticides, 18 herbicides, three fungicides, and 40 pesticide degradates. Data from all sites combined show that 7 of the 10 most frequently detected pesticides were herbicides, with atrazine $(70 \%)$ and metolachlor $(83 \%)$ detected at every site. Dacthal, acetochlor, simazine, alachlor, and pendimethalin were detected in more than $50 \%$ of the samples. Chlorpyrifos, carbaryl, and diazinon were the only insecticides among the 10 most frequently detected compounds. Of the remaining pesticide parent compounds, 18 were detected in fewer than $30 \%$ of the samples, and 13 were not detected. The most frequently detected degradates were deethylatrazine; the oxygen analogs (OAs) of the organophosphorus insecticides chlorpyrifos, diazinon, and malathion; and 1-napthol (degradate of carbaryl). Deethylatrazine was detected in nearly $70 \%$ of the samples collected in Maryland, Indiana, and Nebraska but was detected only once in California. The OAs of chlorpyrifos and diazinon were detected primarily in California. Degradates of the acetanilide herbicides were rarely detected in rain, indicating that they are not formed in the atmosphere or readily volatilized from soils. Herbicides accounted for 91 to $98 \%$ of the total pesticide mass deposited by rain except in California, where insecticides accounted for $61 \%$ in 2004. The mass of pesticides deposited by rainfall was estimated to be less than $2 \%$ of the total applied in these agricultural areas.

Copyright $\odot 2008$ by the American Society of Agronomy, Crop Science Society of America, and Soil Science Society of America. All rights reserved. No part of this periodical may be reproduced or transmitted in any form or by any means, electronic or mechanical, including photocopying, recording, or any information storage and retrieval system, without permission in writing from the publisher.

Published in J. Environ. Qual. 37:1101-1115 (2008).

doi:10.2134/jeq2007.0079

Received 12 Feb. 2007.

*Corresponding author (jrvogel@usgs.gov).

○ ASA, CSSA, SSSA

677 S. Segoe Rd., Madison, WI 53711 USA
$\mathrm{M}$ ORE than 200 agricultural chemicals (Thelin and Gianessi, 2000) are used each year in the production of food and fiber. These chemicals include natural elements such as sulfur, compounds based on naturally occurring products such as pyrethroid insecticides, and synthetic chemicals such as organophosphorus insecticides and triazine herbicides. Pesticide application methods vary and can strongly affect how much of the applied chemical reaches the target site and how much escapes into the atmosphere (Majewski and Capel, 1995). Pesticides also have the potential to enter the atmosphere through volatilization from soil and plant surfaces and through wind erosion (Bedos et al., 2002). Once in the atmosphere, a pesticide may partition between the gas and particle phases, undergo photochemical transformation reactions, be transported downwind, and perhaps return to the Earth's surface by wet (rain, snow) and dry (particle settling or impaction) depositional processes. Rainfall is an efficient mechanism for cleansing the atmosphere of these pollutants through the processes of rainout and washout (Bidleman, 1988, 1999; Majewski and Capel, 1995). Rainout occurs when droplets acquire the contaminant from within the cloud as water vapor condenses around particles or aerosols or by the gas-phase material partitioning into the coalesced water droplet. Washout occurs when the falling droplets intercept and scavenge particles and gas-phase material below the cloud as they fall.

The pesticide content in rain has been studied in a number of environments around the world and has been reviewed through 1992 and 1998, respectively, by Majewski and Capel (1995) and Van Dijk and Guicherit (1999). Many other studies in North America have been reported recently (Miller et al., 2000; Thurman and Cromwell, 2000; Park et al., 2001, 2002; Carlson et al., 2004; Aulagnier and Poissant, 2005; Goel et al., 2005; Waite et al., 2005), as have studies in Europe (Charizopoulos and Papadopoulou-Mourkidou, 1999; Polkowska et al., 2000; De Rossi et al., 2003; Asman et al., 2005).

This paper compares the spatial and temporal patterns of the occurrence of a large number of current-use pesticides and selected degradates in rain during two growing seasons at four locations across the USA (Capel et al., 2008). Study locations in the East and Midwest (Maryland, Indiana, Nebraska) are dominated by corn and soybean row crops, whereas cropland in the West (California) is dominated by almonds and vineyards with some corn and dairy. A more complete description of the watersheds can be found in Capel et al. (2008). The target chemicals ( 42 parent pesticides and 40 degradates) represent broad spectrums of pesticide classes, physical/chemical properties,

J.R. Vogel, U.S. Geological Survey, 5231 S. 19th St., Lincoln, NE 68512. M.S. Majewski, U.S. Geological Survey, Placer Hall-6000 J St., Sacramento, CA 95819. P.D. Capel, U.S Geological Survey, 122 CivEng. Bldg., 500 Pillsbury Dr. SE, Minneapolis, MN 55455.

Abbreviations: DEA, deethylatrazine; OA, oxygen analog. 
usage amounts, and application rates and methods. This is the first study of current-use pesticides in rain that includes a large number of degradates as target chemicals. For each of the studied watersheds, the loads of individual pesticides in the rain were calculated and compared with the estimated application amounts of the same pesticides in the watersheds at three different scales (local, intermediate, and broad) to illustrate the importance of the atmosphere in the overall mass transport of pesticides in the environment.

\section{Materials and Methods}

Rainfall samples were collected at single locations in small, well defined, intensively agricultural watersheds in Maryland, Indiana, Nebraska, and California during the 2003 and 2004 growing seasons. The specific sampling location in each watershed was chosen to meet the criteria of the National Atmospheric Deposition Program (Bigelow et al., 2001). The rainfall samples were collected using modified AeroChemetrics (Bushnel, FL) samplers, which collected wet deposition only. The modifications included collecting the rainfall in a Teflon-lined bucket that drained into a clean, glass carboy held in a refrigerator below the rain sampler. The rain was collected as a weekly integrated sample during the months of March through August or September, except in California, where rain was collected on an individual-event basis (primarily during December through March) because of the infrequency of rainfall. Weekly integrated samples were used at some sites for this project to obtain larger sample volumes (as opposed to single storm events) and to cut down on analytical costs on sites that have many storm events. As a result, the resolution of looking at individual storms was lost, and there was a potential for volatilization during the week-long refrigerated storage on site. Compounds with a Henry's Law constant of $<10^{-2}$ atm $\mathrm{m}^{3} /$ mole, however, lose $<1 \%$ of their mass through volatilization in $7 \mathrm{~d}$ (which includes all compounds analyzed in this study) (Capel and Larson, 1995). Biological degradation in the refrigerated rainfall samples was assumed to be negligible. A fully instrumented meteorological station was co-located with the rain sampler to quantify the meteorological conditions, including rainfall amounts, during the study.

Rain samples were filtered through a Supor (Pall Corporation, East Hills, NY) membrane filter $(0.45 \mu \mathrm{m}$ pore diameter $)$, split, and transferred to amber glass bottles that were shipped on ice overnight to USGS laboratories for analysis. The pesticides were isolated from the water by solid-phase extraction and analyzed using gas chromatography-mass spectrometry and high-performance liquid chromatography-mass spectrometry. The target chemicals included 21 insecticides, 18 herbicides, three fungicides, and 40 degradates (see Capel et al. [2008] for a complete listing of parent and degradate pesticides and selected pesticide properties). The surrogates diazinon- $\mathrm{d} 10$ and alpha-HCH-d6 were spiked into each rain sample at the laboratory, with mean recoveries of $102 \%$ and $90 \%$, respectively. More complete details on the sampling, analytical, and qualitycontrol methods can be found in Capel et al. (2008).

Pesticide use estimates were compiled for each of the study areas for three different areal scales (Capel et al., 2008). The areas comprising the three different scales in Indiana, Nebraska, and
California corresponded to nested watersheds surrounding the sampling site and were deemed appropriate to represent pesticide use because of the consistent agricultural land uses in these areas. In Maryland, county-level and multiple county-level pesticide use represented the intermediate- and broad-scale areas because of the varied land-use patterns at larger scales. At the Eastern and Midwestern sites (Maryland, Indiana, and Nebraska), pesticide use in the local-scale watershed $\left(1.5-33 \mathrm{~km}^{2}\right)$ was estimated by interviewing the individual farmers about the crops they planted and what pesticides they applied and when during 2003 and 2004. Pesticide use in the intermediate scale (about 250-1000 $\mathrm{km}^{2}$ ) and broad scale (about 500-30,000 $\mathrm{km}^{2}$ ) watersheds that encompassed the sampling locations was estimated using the 1997 county-wide use estimates (Thelin and Gianessi, 2000) for Maryland, Indiana, and Nebraska. In Maryland, pesticide use for Kent County was used for the intermediate-scale data, and the summed pesticide use for Harford, Baltimore, Cecil, Kent, and Queen Anne's Counties were used for the broad-scale data. Although pesticide use patterns may have changed and many new pesticides may have been introduced between 1997 and the timing of this study, the county-wide estimates represent the most recent widespread pesticide use data available. These data were based on average usage for different crop types within an area and may not necessarily represent agricultural practices at a specific location. For California, the pesticide use estimates were obtained from the California Department of Pesticide Regulation pesticide use database (California Department of Pesticide Regulation, 2004). Because the upper part of the Merced River basin is forested, only the lower part downstream from McSwain Dam was used for calculating the intermediate-scale pesticide use data. More complete details on the pesticide use can be found in Capel et al. (2008).

\section{Results and Discussion}

\section{Pesticide Occurrence and Concentrations}

Table 1 shows the overall frequency of detection for pesticide parent compounds from all four sites along with their national use ranking (among registered active ingredients, both organic and inorganic) for the target parent pesticides in 2003 and 2004. With a few exceptions, pesticides detected in 2003 also were detected in 2004 . The 10 most frequently detected pesticides in rainfall were detected at every sampling site across the country at frequencies ranging from $83 \%$ for metolachlor to $34 \%$ for diazinon. Seventeen compounds were detected in fewer than $30 \%$ of the samples, and 13 compounds were not detected.

Herbicides were the most frequently detected type of pesticide. Two herbicides (metolachlor and atrazine) were detected at every site and were usually among the three most frequently detected pesticides in rain at the sites. Four other herbicides (acetochlor, alachlor, pendimethalin, and simazine) were consistently detected. The herbicide dacthal also was among the 10 pesticides most frequently detected at all sites, but usually at low concentrations. Dacthal is used on many types of vegetable crops, nonagricultural ornamental plants, and cotton. 
Table 1. Frequency of detection of pesticide parent compounds in 2003 and 2004 from all four sites combined and their national use ranking (among registered active ingredients, both organic and inorganic).

\begin{tabular}{|c|c|c|c|c|c|c|c|}
\hline $\begin{array}{l}\text { Pesticide } \\
\text { (type, total number of samples) } \dagger\end{array}$ & $\begin{array}{l}\text { Laboratory } \\
\text { reporting limit } \neq\end{array}$ & $\begin{array}{c}2003 \\
\text { sample }\end{array}$ & $\begin{array}{c}2004 \\
\text { sample }\end{array}$ & $\begin{array}{c}2003+2004 \\
\text { sample }\end{array}$ & $\begin{array}{c}\text { National stream } \\
\text { detection frequency } \\
(1991-2001) \S\end{array}$ & $\begin{array}{c}\text { National } \\
\text { pesticide use } \\
\text { ranking (1997)ף } \\
\end{array}$ & $\begin{array}{c}\text { National pesticide } \\
\text { use ranking (1997)\# } \\
\text { among analyzed } \\
\text { pesticides }\end{array}$ \\
\hline & $\mu g \mathrm{~L}^{-1}$ & & - $\%$ detect & $s-$ & & & \\
\hline Metolachlor $(H, n=108)$ & 0.013 & 90 & 77 & 83 & 83 & 4 & 2 \\
\hline Atrazine $(H, n=107)$ & 0.007 & 69 & 71 & 70 & 80 & 3 & 1 \\
\hline Dacthal $(H, n=108)$ & 0.003 & 67 & 68 & 68 & 11 & 103 & 26 \\
\hline Chlorpyrifos $(\mathrm{I}, n=107)$ & 0.005 & 63 & 66 & 64 & 11 & 17 & 7 \\
\hline Acetochlor $(H, n=106)$ & 0.006 & 51 & 60 & 56 & 31 & 10 & 3 \\
\hline Simazine $(H, n=108)$ & 0.005 & 63 & 51 & 56 & 57 & 34 & 12 \\
\hline Alachlor $(H, n=106)$ & 0.005 & 42 & 57 & 50 & 37 & 15 & 6 \\
\hline Pendimethalin $(H, n=108)$ & 0.022 & 45 & 53 & 49 & 6.6 & 12 & 4 \\
\hline Carbaryl $(\mathrm{I}, n=108)$ & 0.041 & 37 & 37 & 37 & 9.2 & 37 & 13 \\
\hline Diazinon $(\mathrm{l}, n=108)$ & 0.005 & 45 & 25 & 34 & 13 & 81 & 22 \\
\hline Trifluralin $(H, n=108)$ & 0.009 & 33 & 37 & 31 & 13 & 13 & 5 \\
\hline Malathion $(I, n=108)$ & 0.027 & 27 & 21 & 24 & 5.0 & 30 & 11 \\
\hline Prometon $(H, n=107)$ & 0.005 & 16 & 24 & 20 & 43 & NR & NR \\
\hline Iprodione $(F, n=108)$ & 1.42 & 20 & 16 & 18 & NA & 91 & 24 \\
\hline Myclobutanil (F, $n=108)$ & 0.008 & 16 & 18 & 17 & NA & 153 & 34 \\
\hline Methidathion $(\mathrm{I}, n=108)$ & 0.006 & 12 & 9 & 10 & NA & 126 & 30 \\
\hline Azinphos-methyl $(\mathrm{I}, n=108)$ & 0.05 & 6 & 9 & 7 & 1.3 & 56 & 16 \\
\hline Phosmet $(\mathrm{I}, n=101)$ & 0.008 & 8 & 6 & 7 & NA & 67 & 19 \\
\hline Dimethanamid $(\mathrm{H}, n=52)$ & 0.02 & 0 & 6 & 6 & NA & 28 & 9 \\
\hline Prometryn $(H, n=108)$ & 0.005 & 6 & 7 & 6 & NA & 63 & 18 \\
\hline Dichlorvos $(I, n=108)$ & 0.012 & 2 & 7 & 5 & NA & NR & NR \\
\hline Propachlor $(\mathrm{H}, n=43)$ & 0.025 & 0 & 13 & 5 & 1.5 & 77 & 21 \\
\hline Methyl parathion $(\mathrm{I}, n=108)$ & 0.015 & 4 & 4 & 4 & 1.0 & 29 & 10 \\
\hline Flufenacet $(\mathrm{H}, n=52)$ & 0.02 & 0 & 4 & 4 & NA & NR & NR \\
\hline Pronamide $(H, n=108)$ & 0.004 & 0 & 5 & 3 & 2.2 & 143 & 31 \\
\hline Terbuthylazine $(H, n=108)$ & 0.010 & 2 & 2 & 2 & NA & NR & NR \\
\hline Metribuzin $(H, n=108)$ & 0.006 & 0 & 2 & 1 & 18 & 43 & 14 \\
\hline Benfluralin $(H, n=108)$ & 0.01 & 0 & 2 & 1 & 0.8 & NR & NR \\
\hline Fipronil $(\mathrm{I}, n=108)$ & 0.016 & 0 & 2 & 1 & NA & NR & NR \\
\hline Terbufos $(\mathrm{l}, n=108)$ & 0.017 & 0 & 0 & 0 & 0.1 & 27 & 8 \\
\hline Phorate $(\mathrm{I}, n=108)$ & 0.011 & 0 & 0 & 0 & 0.1 & 44 & 15 \\
\hline Dimethoate $(I, n=108)$ & 0.006 & 0 & 0 & 0 & NA & 60 & 17 \\
\hline Permethrin $(\mathrm{I}, n=108)$ & 0.006 & 0 & 0 & 0 & 0.2 & 71 & 20 \\
\hline Fenamiphos $(\mathrm{I}, n=108)$ & 0.029 & 0 & 0 & 0 & NA & 89 & 23 \\
\hline Metalaxyl $(I, n=108)$ & 0.005 & 0 & 0 & 0 & NA & 95 & 25 \\
\hline Ethion $(I, n=108)$ & 0.004 & 0 & 0 & 0 & NA & 109 & 27 \\
\hline Fonofos $(\mathrm{I}, n=108)$ & 0.003 & 0 & 0 & 0 & 3.1 & 114 & 28 \\
\hline Dicrotophos $(\mathrm{I}, n=108)$ & 0.084 & 0 & 0 & 0 & NA & 117 & 29 \\
\hline Cypermethrin $(I, n=108)$ & 0.009 & 0 & 0 & 0 & NA & 146 & 32 \\
\hline Cyfluthrin $(\mathrm{I}, n=108)$ & 0.008 & 0 & 0 & 0 & NA & 151 & 33 \\
\hline Tebuthiuron $(I, n=108)$ & 0.016 & 0 & 0 & 0 & 20 & 164 & 35 \\
\hline Isofenphos $(I, n=108)$ & 0.003 & 0 & 0 & 0 & NA & NR & NR \\
\hline
\end{tabular}

$\uparrow \mathrm{H}$, herbicide; I, insecticide; F, fungicide; n, total number of rain samples collected during 2003-2004; NR, not ranked; NA, not available.

₹ All reported concentrations, even those less than the laboratory reporting limit (LRL), were used for calculating the summary statistics and detection frequencies. The LRLs of the different pesticides are not all the same and make the direct comparisons of detection frequencies of different pesticides somewhat ambiguous. Using a common LRL for all pesticides creates a substantial loss of information.

$\S$ From Martin et al. (2002).

I Out of 220; from Thelin and Gianessi (2000).

\# Of the 34 that were analyzed and that were in the rankings; from Thelin and Gianessi (2000).

Chlorpyrifos, carbaryl, and diazinon were the most frequently detected insecticides during this study. Of the pesticides analyzed, the top seven most used compounds nationally were also among the 11 most frequently detected in rain.

\section{Maryland}

Agricultural products in the Maryland study area were a mixture of dairy, corn, sweet corn, soybean, and alfalfa. Metolachlor, atrazine, and simazine were the only herbicides reportedly used in 


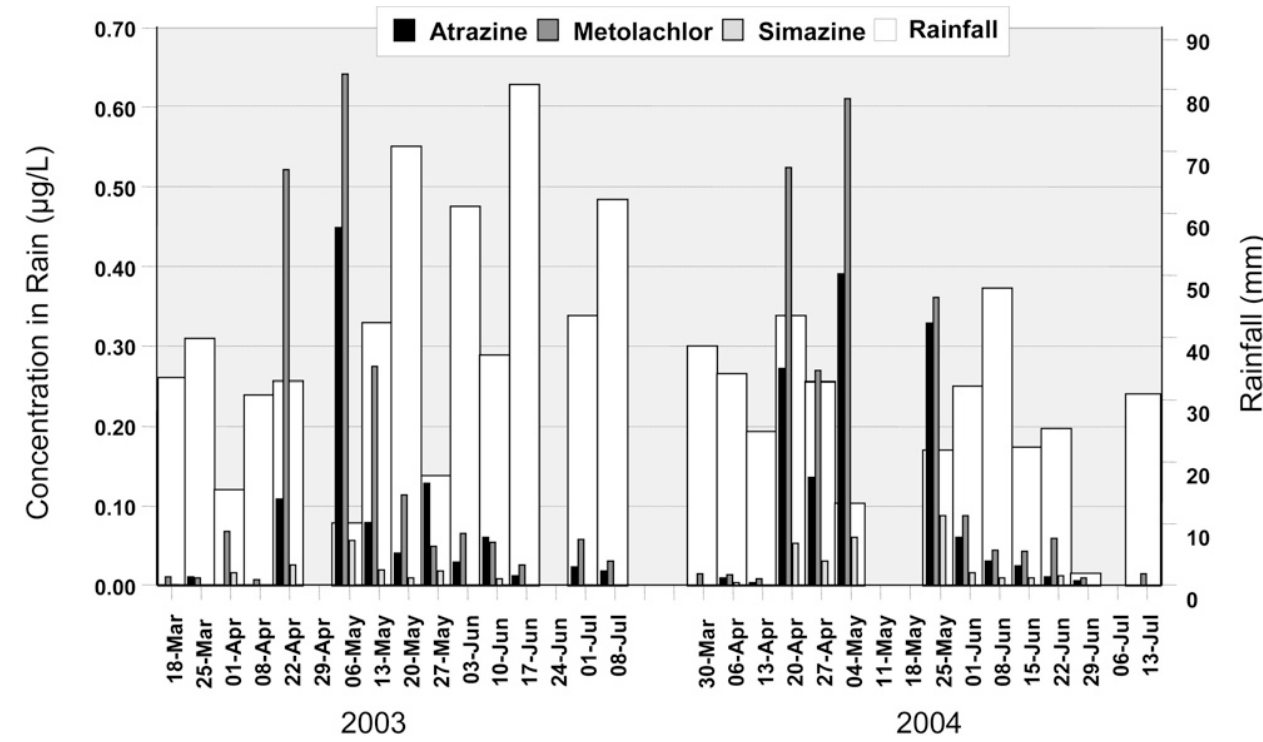

Fig. 1. Atrazine, metolachlor, and simazine concentrations in weekly composite rain samples, plus weekly rainfall amounts, at the rain sampling site in Maryland during the growing season in 2003 and 2004. of each year, concurrent with the planting of crops in the area.

Dacthal had no reported agricultural use in the local-scale area and only minimal agricultural use (0.15 $\mathrm{Mg} \mathrm{yr}^{-1}$ was reported in the broad-scale area, but there may have been unreported usage in commercial nurseries near the study site). Dacthal was detected in $74 \%$ of the samples but at low concentrations (maximum: $0.009 \mu \mathrm{g} \mathrm{L}^{-1}$; median: $0.002 \mu \mathrm{g} \mathrm{L}^{-1}$ [estimated]; laboratory reporting limit $=0.003 \mu \mathrm{g} \mathrm{L}^{-1}$ ) (Tables 1 and 2A).

Indiana

The primary crops in the Indiana study area were corn, soybeans, and alfalfa. The rain sampling site was in an agricultural area on a fence-line between a the immediate area around the rain sampler (Capel et al., 2008). Metolachlor was detected in every sample, with the largest concentrations occurring during late April through the end of May, concurrent with the planting of corn and soybeans (Fig. 1; Table 2A). This same seasonal pattern occurred for atrazine and simazine. The concentrations of simazine in rain were much lower than those of metolachlor or atrazine despite similar reported use amounts of these three herbicides in the local-scale watershed. This can be explained primarily by the vapor pressure of simazine $\left(2.95 \times 10^{-6} \mathrm{~Pa}\right.$ at $\left.25^{\circ} \mathrm{C}\right)$, which is three orders of magnitude lower than metolachlor's $\left(4.19 \times 10^{-3} \mathrm{~Pa}\right.$ at $\left.25^{\circ} \mathrm{C}\right)$ and one order of magnitude less than atrazine's $\left(3.85 \times 10^{-5} \mathrm{~Pa}\right.$ at $\left.25^{\circ} \mathrm{C}\right)$.

With few exceptions, the metolachlor concentrations in rain were greater than those of atrazine, even though the use of atrazine was greater than metolachlor at the local scale. Because the vapor pressure of metolachlor is two orders of magnitude higher than that of atrazine, more of the applied metolachlor volatilizes into the lower atmosphere. In addition, metolachlor use on the intermediate- and broad-scale watersheds was nearly twice that of atrazine, so atmospheric transport of all these herbicides from the surrounding areas into the sampling area cannot be discounted as a factor favoring metolachlor concentrations in the rain samples. Glotfelty et al. (1990) showed that alachlor and metolachlor were transient in the atmosphere and susceptible to rapid vapor-phase degradation and that atrazine and simazine were long-lived in the atmosphere and susceptible to regional- and long-range atmospheric transport.

Three other herbicides used on corn and soybeans - pendimethalin, acetochlor, and alachlor — were detected in the rainfall. Although no applications were reported in the local-scale watershed during the study, all three of these herbicides were used on the intermediate- and broad-scale watersheds. The maximum concentrations for all of these herbicides followed the same temporal trends as were seen with metolachlor, atrazine, and simazine, with the highest concentrations occurring in April and May corn field and a soybean field (rotated oppositely in each field). Pesticide use in the local-scale watershed in 2003 consisted primarily of atrazine $(245 \mathrm{~kg})$ and chlorpyrifos $(103 \mathrm{~kg})$ with minor amounts of metolachlor $(5 \mathrm{~kg})$ and acetochlor $(3 \mathrm{~kg})$. None of these and none of the other targeted pesticides that were analyzed was reportedly used in the local-scale area in 2004.

The herbicides atrazine, acetochlor, and metolachlor were detected in $>80 \%$ of all samples (Table $2 \mathrm{~B}$ ) and in every sample collected in 2003. The greatest concentrations were detected between mid-April and mid-June, which corresponded to the period of application (Fig. 2; Table 2B).

In 2003, acetochlor use in the local-scale watershed was much less than atrazine use, and the median acetochlor concentration was about half that of atrazine in 2003 (0.069 and $0.16 \mu \mathrm{g} \mathrm{L}{ }^{-1}$, respectively). During the two time periods in 2003 when the greatest atrazine concentrations occurred, the corresponding acetochlor concentrations were also the highest. The acetochlor concentrations during the rest of both seasons were nearly equivalent to those of atrazine, but the concentrations decreased more rapidly in June and July (Fig. 2), as would be expected because acetochlor, similar to alachlor, has a shorter atmospheric residence time than does atrazine. In 2004, atrazine and acetochlor were frequently detected at nearly the same concentrations as in 2003 despite no reported use in the local-scale area. Metolachlor concentrations and detection frequencies followed the same temporal pattern as atrazine and acetochlor, but the maximum and median concentrations were considerably less. The reported use for metolachlor in the local-scale watershed in 2003 was low $(5 \mathrm{~kg})$ and similar to that of acetochlor. For the intermediate- and broad-scale watersheds, metolachlor was widely used and nearly equivalent to atrazine. The use of all three of these herbicides outside the local-scale watershed made a substantial contribution to the observed concentrations of these herbicides in rain, especially in 2004. 
Table 2. Statistical summary of pesticides in rain samples at four sites (in order of percent detections; number of samples shown in parentheses is the total for the 2-yr study period).

\begin{tabular}{|c|c|c|c|c|c|c|c|c|c|c|}
\hline \multirow[b]{2}{*}{ Pesticide } & \multirow{2}{*}{$\begin{array}{c}\% \\
\text { Detects }\end{array}$} & \multirow[b]{2}{*}{$>0.01 \mu \mathrm{g} \mathrm{L}^{-1}$} & \multirow[b]{2}{*}{$>0.1 \mu \mathrm{g} \mathrm{L}^{-1}$} & \multirow[b]{2}{*}{$>1.0 \mu \mathrm{g} \mathrm{L}^{-1}$} & \multirow{2}{*}{$\begin{array}{c}\text { Max } \\
\text { concentration }\end{array}$} & \multirow{2}{*}{$\begin{array}{c}\text { Median } \\
\text { concentration }\end{array}$} & \multirow{2}{*}{$\begin{array}{c}\text { Date of } \\
\text { maximum } \\
\text { concentration }\end{array}$} & \multicolumn{2}{|c|}{ Wet deposition } & \multirow{2}{*}{$\begin{array}{c}\text { Date of } \\
\text { maximum } \\
\text { deposition }\end{array}$} \\
\hline & & & & & & & & 2003 & 2004 & \\
\hline & & & -\% & & $-\mu \mathrm{g}$ & $\mathrm{L}^{-1}$ & & $-\mu \mathrm{g}$ & $\mathrm{m}^{-2}-$ & \\
\hline \multicolumn{11}{|l|}{ A. Maryland } \\
\hline Metolachlor $(n+=27)$ & 100 & E93 & 30 & 0 & 0.643 & 0.055 & 6 May 2003 & 59.4 & 57.0 & 20 Apr. 2004 \\
\hline Atrazine $(n=27)$ & 81 & 74 & 26 & 0 & 0.450 & 0.026 & 6 May 2003 & 24.6 & 33.6 & 20 Apr. 2004 \\
\hline Dacthal $(n=27)$ & 74 & 0 & 0 & 0 & 0.009 & E0.002 & 1 June 2004 & 0.840 & 1.10 & 8 June 2004 \\
\hline Simazine $(n=27)$ & 63 & 52 & 0 & 0 & 0.089 & 0.010 & 25 May 2004 & 4.08 & 7.91 & 20 Apr. 2004 \\
\hline Pendimethalin $(n=27)$ & 59 & E56 & 4 & 0 & 0.111 & E0.020 & 25 May 2004 & 11.6 & 9.46 & 20 May 2003 \\
\hline Deethyl atrazine $(n=27)$ & 59 & 30 & 0 & 0 & 0.057 & 0.006 & 6 May 2003 & 2.63 & 3.79 & 25 May 2004 \\
\hline Carbaryl $(n=27)$ & 48 & NA & 0 & 0 & E0.026 & $<0.041$ & 4 May 2004 & 2.15 & 2.28 & 8 July 2003 \\
\hline Alachlor $(n=27)$ & 44 & 11 & 0 & 0 & 0.038 & $<0.005$ & 25 May 2004 & 1.03 & 2.80 & 25 May 2004 \\
\hline Chlorpyrifos $(n=27)$ & 44 & 0 & 0 & 0 & 0.009 & $<0.005$ & 1 June 2004 & 0.929 & 1.12 & 17 June 2003 \\
\hline Acetochlor $(n=27)$ & 41 & 33 & 7 & 0 & 0.173 & $<0.006$ & 4 May 2004 & 7.59 & 11.7 & 20 Apr. 2004 \\
\hline Diazinon $(n=27)$ & 19 & 0 & 0 & 0 & 0.008 & $<0.005$ & 1 June 2004 & 0.365 & 0.563 & 1 June 2004 \\
\hline Azinphos-methyl $(n=27)$ & 15 & NA & 0 & 0 & E0.025 & $<0.050$ & 8 June 2004 & 0.740 & 1.72 & 8 June 2004 \\
\hline Prometon $(n=27)$ & 11 & NA & 0 & 0 & E0.012 & $<0.015$ & 15 June 2004 & 0.336 & 0.472 & 8 July 2003 \\
\hline Phosmet $(n=26)$ & 4 & 4 & 0 & 0 & 0.011 & $<0.008$ & 8 June 2004 & 0 & 0.528 & 8 June 2004 \\
\hline Dimethenamid $(n=12)$ & 4 & NA & 0 & 0 & 0.04 & $<0.02$ & 4 May 2004 & 0 & 0.528 & 4-May-2004 \\
\hline Chlorpyrifos OA $(n=27)$ & 4 & NA & 0 & 0 & E0.006 & $<0.056$ & 6 Apr. 2004 & 0 & 0.191 & 6-Apr-2004 \\
\hline Myclobutanil $(n=27)$ & 4 & 0 & 0 & 0 & E0.007 & $<0.008$ & 27 Apr. 2004 & 0 & 0.233 & 27 Apr. 2004 \\
\hline \multirow[t]{5}{*}{ Trifluralin $(n=27)$} & 4 & 0 & 0 & 0 & E0.004 & $<0.009$ & 30 Mar. 2004 & 0 & 0.147 & 30 Mar. 2004 \\
\hline & & & & & \multicolumn{2}{|c|}{ Sum of all herbicides analyzed } & & 112.1 & 128.5 & 20 Apr. 2004 \\
\hline & & & & & \multicolumn{2}{|c|}{ Sum of all insecticides analyzed } & & 4.18 & 6.41 & 8 June 2004 \\
\hline & & & & & \multicolumn{2}{|c|}{ Sum of all fungicides analyzed } & & 0 & 0.233 & 27 Apr. 2004 \\
\hline & & & & & \multicolumn{3}{|c|}{ Sum of all pesticides analyzed } & 116.3 & 135.1 & 20 Apr. 2004 \\
\hline \multicolumn{11}{|l|}{ B. Indiana } \\
\hline Atrazine $(n=25)$ & 88 & 84 & 52 & 12 & 6.58 & 0.014 & 27 May 2003 & 239 & 72.7 & 27 May 2003 \\
\hline Acetochlor $(n=25)$ & 84 & 72 & 32 & 12 & 1.98 & 0.028 & 13 May 2003 & 97.5 & 55.3 & 27 May 2003 \\
\hline Metolachlor $(n=26)$ & 81 & E62 & 31 & 0 & 0.558 & 0.017 & 27 Apr. 2004 & 34.0 & 21.0 & 27 May 2003 \\
\hline Deethyl atrazine $(n=26)$ & 73 & 54 & 12 & 0 & 0.339 & 0.012 & 27 May 2003 & 23.1 & 8.44 & 29 Apr. 2003 \\
\hline Prometon $(n=25)$ & 64 & E16 & 0 & 0 & E0.0122 & E0.008 & 24 June 2003 & 0.827 & 1.56 & 6 May 2003 \\
\hline Alachlor $(n=26)$ & 58 & 42 & 0 & 0 & 0.050 & 0.014 & 27 May 2003 & 6.03 & 2.60 & 27 May 2003 \\
\hline Malathion $(n=26)$ & 54 & E46 & 0 & 0 & 0.087 & E0.014 & 24 June 2003 & 4.27 & 7.59 & 1 July 2003 \\
\hline Chlorpyrifos $(n=26)$ & 54 & 31 & 4 & 0 & 0.110 & 0.005 & 27 May 2003 & 6.56 & 0.811 & 1 July 2003 \\
\hline Simazine $(n=26)$ & 46 & 31 & 0 & 0 & 0.037 & $<0.005$ & 27 May 2003 & 3.62 & 1.62 & 18 May 2004 \\
\hline Malathion OA $(n=22)$ & 36 & 36 & 5 & 0 & 0.308 & $<0.008$ & 17 Aug. 2004 & 4.97 & 12.3 & 8 June 2004 \\
\hline Diazinon $(n=26)$ & 31 & 0 & 0 & 0 & 0.010 & $<0.005$ & 15 July 2003 & 1.45 & 0.253 & 8 July 2003 \\
\hline Dacthal $(n=26)$ & 31 & 0 & 0 & 0 & 0.005 & $<0.003$ & 18 May 2004 & 0.168 & 0.245 & 18 May 2004 \\
\hline Pendimethalin $(n=26)$ & 31 & NA & 4 & 0 & 0.484 & $<0.022$ & 29 Apr. 2003 & 28.9 & 1.55 & 8 June 2004 \\
\hline Carbaryl $(n=26)$ & 25 & NA & 0 & 0 & E0.034 & $<0.041$ & 15 July 2003 & 5.72 & 0.999 & 1 July 2003 \\
\hline Phosmet $(n=25)$ & 20 & 20 & 0 & 0 & 0.047 & $<0.008$ & 13 May 2003 & 1.74 & 1.07 & 18 May 2004 \\
\hline 1-Naphthol $(n=25)$ & 17 & NA & 0 & 0 & 0.014 & $<0.088$ & 29 July 2003 & 1.03 & 0 & 10 June 2003 \\
\hline Dimethenamid $(n=12)$ & 17 & NA & 0 & 0 & 0.04 & $<0.02$ & 27 Apr. 2004 & 0 & 1.13 & 27 Apr. 2004 \\
\hline Azinphos-methyl $(n=26)$ & 15 & 12 & 0 & 0 & 0.082 & $<0.050$ & 29 July 2003 & 1.44 & 2.49 & 6 May 2003 \\
\hline Chlorpyrifos OA $(n=26)$ & 8 & NA & 0 & 0 & 0.014 & $<0.056$ & 27 May 2003 & 0.683 & 0 & 29 July 2003 \\
\hline Acetochlor ESA $(n=23)$ & 4 & NA & 0 & 0 & 0.05 & $<0.05$ & 29 July 2003 & 0.565 & 0 & 29 Apr. 2003 \\
\hline Alachlor ESA $(n=23)$ & 4 & NA & 0 & 0 & 0.02 & $<0.05$ & 6 July 2004 & 0 & 0.049 & 6 July 2004 \\
\hline Terbuthylazine $(n=26)$ & 4 & 0 & 0 & 0 & 0.009 & $<0.010$ & 27 May 2003 & 0.192 & 0 & 27 May 2003 \\
\hline sTrifluralin $(n=26)$ & 4 & 0 & 0 & 0 & 0.002 & $<0.009$ & 24 Aug. 2004 & 0.096 & 0 & 24 Aug. 2004 \\
\hline & & & & & Sum of all herbi & icides analyzed & & 434 & 166 & 27 May 2003 \\
\hline & & & & & Sum of all insec & ticides analyzed & & 27.9 & 25.5 & 17 Aug. 2004 \\
\hline & & & & & Sum of all fungi & icides analyzed & & 0 & 0 & not detected \\
\hline & & & & & Sum of all pesti & cides analyzed & & 462 & 192 & 27 May 2003 \\
\hline
\end{tabular}

(continued)

Three other herbicides that had no reported use in the local-scale watershed but had $>50 \mathrm{Mg} \mathrm{yr}^{-1}$ use in the broad-scale watershed were pendimethalin, alachlor, and dimethenamid. Pendimethalin was detected in only $31 \%$ of the samples, mostly in 2003, and was detected only twice in 2004 (Table 2B). Concentrations of pendimethalin were considerably lower than those of atrazine or acetochlor, with the exception of the 29 Apr. 2003 sample $\left(0.48 \mu \mathrm{g} \mathrm{L}^{-1}\right)$. Because pendime- 


\begin{tabular}{|c|c|c|c|c|c|c|c|c|c|c|}
\hline \multirow[b]{2}{*}{ Pesticide } & \multirow{2}{*}{$\begin{array}{c}\% \\
\text { Detects }\end{array}$} & \multirow{2}{*}{$>0.01 \mu \mathrm{g} \mathrm{L}^{-1}$} & \multirow[b]{2}{*}{$>0.1 \mu \mathrm{g} \mathrm{L}^{-1}$} & \multirow[b]{2}{*}{$>1.0 \mu \mathrm{g} \mathrm{L}^{-1}$} & \multirow{2}{*}{$\begin{array}{c}\text { Max } \\
\text { concentration }\end{array}$} & \multirow{2}{*}{$\begin{array}{c}\text { Median } \\
\text { concentration }\end{array}$} & \multirow{2}{*}{$\begin{array}{c}\text { Date of } \\
\text { maximum } \\
\text { concentration }\end{array}$} & \multicolumn{2}{|c|}{ Wet deposition } & \multirow{2}{*}{$\begin{array}{c}\text { Date of } \\
\text { maximum } \\
\text { deposition }\end{array}$} \\
\hline & & & & & & & & 2003 & 2004 & \\
\hline & 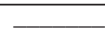 & & $\%$ & +2 & $\mu g \mathrm{~L}$ & $\mathrm{~L}^{-1}$ & & $-\mu \mathrm{g}$ & $\mathrm{m}^{-2}$ & \\
\hline \multicolumn{11}{|l|}{ C. Nebraska } \\
\hline Atrazine $(n=32)$ & 94 & 81 & 44 & 13 & 19.0 & 0.053 & 13 Apr. 2004 & 83.2 & 289 & 4 May 2004 \\
\hline Metolachlor $(n=32)$ & 88 & E63 & 25 & 3 & 1.76 & 0.014 & 13 Apr. 2004 & 24.5 & 58.7 & 18 May 2004 \\
\hline Acetochlor $(n=31)$ & 87 & 77 & 39 & 13 & 4.39 & 0.041 & 13 Apr. 2004 & 101 & 187 & 4 May 2004 \\
\hline Alachlor $(n=30)$ & 87 & 67 & 17 & 0 & 0.282 & 0.016 & 4 May 2004 & 12.8 & 42.9 & 18 May 2004 \\
\hline Deethyl atrazine $(n=32)$ & 75 & 63 & 9 & 0 & 0.273 & 0.022 & 13 Apr. 2004 & 12.1 & 28.1 & 4 May 2004 \\
\hline Chlorpyrifos $(n=31)$ & 74 & 26 & 3 & 0 & 0.193 & 0.006 & 10 Aug. 2004 & 1.98 & 4.91 & 10 Aug. 2004 \\
\hline Trifluralin $(n=32)$ & 72 & 44 & 3 & 0 & 0.148 & E0.008 & 27 May 2003 & 7.65 & 5.76 & 27 May 2003 \\
\hline Dacthal $(n=32)$ & 69 & 0 & 0 & 0 & 0.009 & 0.003 & 5 Oct. 2004 & 0.510 & 1.14 & 25 May 2004 \\
\hline Pendimethalin $(n=32)$ & 34 & NA & 0 & 0 & 0.046 & $<0.022$ & 1 June 2004 & 5.04 & 3.96 & 29 Apr. 2003 \\
\hline Simazine $(n=32)$ & 28 & 6 & 0 & 0 & 0.071 & $<0.005$ & 13 Apr. 2004 & 0.549 & 1.61 & 25 May 2004 \\
\hline Malathion OA $(n=22)$ & 18 & 18 & 0 & 0 & 0.046 & $<0.008$ & 28 Oct. 2003 & 0.278 & 0.681 & 5 Oct. 2004 \\
\hline Carbaryl $(n=32)$ & 16 & NA & 0 & 0 & E0.024 & $<0.041$ & 25 May 2004 & 0.660 & 1.46 & 25 May 2004 \\
\hline Methyl parathion $(n=32)$ & 13 & 13 & 0 & 0 & 0.056 & $<0.006$ & 15 July 2003 & 1.47 & 1.72 & 20 July 2004 \\
\hline Prometryn $(n=32)$ & 13 & 3 & 0 & 0 & 0.013 & $<0.005$ & 11 May 2004 & 0.119 & 0.796 & 18 May 2004 \\
\hline Malathion $(n=32)$ & 13 & NA & 0 & 0 & 0.030 & $<0.027$ & 5 Oct. 2004 & 0.167 & 0.405 & 5 Oct. 2004 \\
\hline Flufenacet $(n=18)$ & 11 & NA & 0 & 0 & 0.05 & $<0.02$ & 4 May 2004 & 0 & 3.09 & 4 May 2004 \\
\hline $\begin{array}{l}\text { 2-Chloro-2,6-diethylacet anilide } \\
(n=32)\end{array}$ & 9 & 0 & 0 & 0 & 0.009 & $<0.005$ & 11 May 2004 & 0 & 0.024 & 25 May 2004 \\
\hline Methyl-paraoxon $(n=32)$ & 6 & NA & 0 & 0 & 0.037 & $<0.030$ & 15 July 2003 & 0.678 & 0 & 29 July 2003 \\
\hline Prometon $(n=31)$ & 6 & NA & 0 & 0 & E0.009 & $<0.015$ & 11 May 2004 & 0 & 0.245 & 4 May 2004 \\
\hline Alachlor ESA $(n=23)$ & 4 & NA & 0 & 0 & E0.02 & $<0.05$ & 6 July 2004 & 0 & 1.18 & 6 July 2004 \\
\hline Chlorpyrifos OA $(n=32)$ & 3 & NA & 0 & 0 & E0.009 & $<0.056$ & 20 July 2004 & 0 & 0.391 & 20 July 2004 \\
\hline Benfluralin $(n=32)$ & 3 & 0 & 0 & 0 & E0.006 & $<0.010$ & 20 July 2004 & 0 & 0.269 & 20 July 2004 \\
\hline Desulfinyl fipronil amide $(n=32)$ & 3 & 3 & 0 & 0 & 0.014 & $<0.009$ & 3 Aug. 2004 & 0 & 0.150 & 3 Aug. 2004 \\
\hline 1-Naphthol $(n=32)$ & 3 & NA & 0 & 0 & E0.006 & $<0.088$ & 29 July 2003 & 0.140 & 0 & 29 July 2003 \\
\hline Diazinon $(n=32)$ & 3 & 0 & 0 & 0 & 0.005 & $<0.005$ & 27 May 2003 & 0.091 & 0 & 27 May 2003 \\
\hline Metribuzin $(n=32)$ & 3 & 3 & 3 & 0 & 0.103 & $<0.006$ & 13 Apr. 2004 & 0 & 0.078 & 13 Apr. 2004 \\
\hline Fipronil $(n=32)$ & 3 & 3 & 0 & 0 & 0.017 & $<0.007$ & 13 Apr. 2004 & 0 & 0.013 & 13 Apr. 2004 \\
\hline Terbuthylazine $(n=32)$ & 3 & 3 & 0 & 0 & 0.014 & $<0.010$ & 13 Apr. 2004 & 0 & 0.011 & 13 Apr. 2004 \\
\hline \multirow[t]{5}{*}{ 3,4-Dichloroaniline $(n=32)$} & 3 & 3 & 0 & 0 & 0.013 & $<0.005$ & 13 Apr. 2004 & 0 & 0.010 & 13 Apr. 2004 \\
\hline & & & & & \multicolumn{3}{|c|}{ Sum of all herbicides analyzed } & 249 & 628 & 4 May 2004 \\
\hline & & & & & \multicolumn{3}{|c|}{ Sum of all insecticides analyzed } & 3.49 & 4.82 & 29 July 2003 \\
\hline & & & & & Sum of all fungi & icides analyzed & & 0 & 0 & not detected \\
\hline & & & & & Sum of all pestic & icides analyzed & & 253 & 633 & 4 May 2004 \\
\hline D. California & & & & & & & & & & \\
\hline Simazine $(n=23)$ & 100 & 100 & 26 & 4 & 15.6 & 0.040 & 12 Mar. 2003 & $114 \ddagger$ & $9.04 \ddagger$ & 12 Mar. 2003 \\
\hline Diazinon $(n=23)$ & 100 & 74 & 22 & 4 & 1.20 & 0.024 & 16 Dec. 2003 & $0.596 \neq$ & $24.1 \neq$ & 16 Dec. 2003 \\
\hline Dacthal $(n=23)$ & 100 & 43 & 0 & 0 & 0.022 & 0.009 & 26 Apr. 2003 & $0.188 \neq$ & $1.19 \neq$ & 29 Jan. 2004 \\
\hline Chlorpyrifos $(n=23)$ & 87 & 65 & 9 & 4 & 1.84 & 0.015 & 24 Feb. 2004 & $0.067 \ddagger$ & $4.88 \neq$ & 16 Dec. 2003 \\
\hline Iprodione $(n=23)$ & 83 & E83 & E39 & E13 & 3.44 & E0.074 & 12 Feb. 2003 & $4.42 \neq$ & $14.9 \neq$ & 24 Feb. 2004 \\
\hline Pendimethalin $(n=23)$ & 78 & E78 & 4 & 0 & 0.143 & E0.021 & 12 Feb. 2003 & $0.121 \neq$ & $3.55 \neq$ & 29 Jan. 2004 \\
\hline Diazinon OA $(n=21)$ & 76 & 57 & 5 & 0 & 0.118 & 0.012 & 16 Dec. 2003 & $0.183 \ddagger$ & $3.69 \neq$ & 16 Dec. 2003 \\
\hline Myclobutanil $(n=23)$ & 74 & 57 & 4 & 0 & 0.113 & 0.014 & 12 Feb. 2003 & $0.862 \ddagger$ & $1.06 \neq$ & 24 Feb. 2004 \\
\hline Chlorpyrifos OA $(n=23)$ & 70 & E52 & 0 & 0 & 0.100 & E0.012 & 24 Feb. 2004 & $0.233 \neq$ & $1.99 \neq$ & 29 Jan. 2004 \\
\hline Carbaryl $(n=23)$ & 70 & E39 & 0 & 0 & 0.093 & E0.020 & 16 Dec. 2003 & $0.529 \neq$ & $1.89 \neq$ & 16 Dec. 2003 \\
\hline Metolachlor $(n=23)$ & 61 & E17 & 4 & 0 & 0.226 & E0.007 & 29 Apr. 2003 & $0.182 \ddagger$ & $0.307 \neq$ & 16 Dec. 2003 \\
\hline Methidathion $(n=23)$ & 48 & 30 & 4 & 0 & 0.691 & $<0.006$ & 16 Dec. 2003 & $0.015 \ddagger$ & $11.1 \neq$ & 16 Dec. 2003 \\
\hline Trifluralin $(n=23)$ & 39 & 4 & 0 & 0 & 0.014 & $<0.009$ & 16 Dec. 2003 & $0.004 \neq$ & $0.602 \ddagger$ & 29 Jan. 2004 \\
\hline Malathion $(n=23)$ & 35 & NA & 0 & 0 & 0.058 & $<0.027$ & 25 Mar. 2004 & $0.270 \neq$ & $0.188 \neq$ & 12 Mar. 2003 \\
\hline 1-Naphthol $(n=23)$ & 30 & NA & 0 & 0 & E0.008 & $<0.088$ & 25 Mar. 2004 & $0 \neq$ & $0.083 \neq$ & 25 Mar. 2004 \\
\hline Propachlor $(n=7)$ & 29 & NA & 29 & 0 & 0.163 & $<0.025$ & 11 Dec. 2003 & 0‡ & $1.89 \neq$ & 11 Dec. 2003 \\
\hline Malathion OA $(n=23)$ & 22 & 22 & 0 & 0 & 0.041 & $<0.008$ & 12 Mar. 2003 & $0.298 \neq$ & $0.146 \neq$ & 12 Mar. 2003 \\
\hline 3,4-Dichloroaniline $(n=23)$ & 22 & 13 & 0 & 0 & 0.033 & $<0.005$ & 16 Dec. 2003 & $0.013 \neq$ & $0.760 \neq$ & 16 Dec. 2003 \\
\hline Dichlorvos $(n=23)$ & 22 & NA & 4 & 0 & 0.517 & $<0.012$ & 16 Feb. 2004 & $0 \neq$ & $0.092 \neq$ & 31 Dec. 2003 \\
\hline Prometryn $(n=23)$ & 13 & 9 & 0 & 0 & 0.031 & $<0.005$ & 11 Dec. 2003 & $0 \neq$ & $0.735 \neq$ & 16 Dec. 2003 \\
\hline Pronamide $(n=23)$ & 13 & 4 & 0 & 0 & 0.017 & $<0.004$ & 25 Mar. 2004 & $0 \neq$ & $0.055 \neq$ & 25 Mar. 2004 \\
\hline
\end{tabular}


Table 2. Cont'd.

\begin{tabular}{|c|c|c|c|c|c|c|c|c|c|c|}
\hline \multirow[b]{2}{*}{ Pesticide } & \multirow{2}{*}{$\begin{array}{c}\% \\
\text { Detects }\end{array}$} & \multirow[b]{2}{*}{$>0.01 \mu \mathrm{g} \mathrm{L}^{-1}$} & \multirow[b]{2}{*}{$>0.1 \mu \mathrm{g} \mathrm{L}^{-1}$} & \multirow[b]{2}{*}{$>1.0 \mu \mathrm{g} \mathrm{L}^{-1}$} & \multirow{2}{*}{$\begin{array}{c}\text { Max } \\
\text { concentration }\end{array}$} & \multirow{2}{*}{$\begin{array}{c}\text { Median } \\
\text { concentration }\end{array}$} & \multirow{2}{*}{$\begin{array}{c}\text { Date of } \\
\text { maximum } \\
\text { concentration }\end{array}$} & \multicolumn{2}{|c|}{ Wet deposition } & \multirow{2}{*}{$\begin{array}{c}\text { Date of } \\
\text { maximum } \\
\text { deposition }\end{array}$} \\
\hline & & & & & & & & \multirow{2}{*}{\multicolumn{2}{|c|}{$\begin{array}{c}2003 \quad 2004 \\
-\mu g m^{-2}-\end{array}$}} & \\
\hline & & & $\%$ & & $\longrightarrow \mathrm{g}$ & $L^{-1}$ & & & & \\
\hline Acetochlor ESA $(n=10)$ & 10 & NA & 0 & 0 & 0.05 & $<0.05$ & 24 Feb. 2004 & $0 \neq$ & $0 \neq$ & NA \\
\hline Alachlor ESA $(n=10)$ & 10 & NA & 0 & 0 & 0.07 & $<0.05$ & 24 Feb. 2004 & $0 \neq$ & $0 \neq$ & NA \\
\hline $\begin{array}{l}\text { 4-Chloro-2-methylphenol } \\
(n=23)\end{array}$ & 9 & 9 & 0 & 0 & 0.059 & $<0.006$ & 12 Feb. 2003 & $0.009 \neq$ & $0 \neq$ & 12 Feb. 2003 \\
\hline Phosmet $(n=18)$ & 6 & 6 & 0 & 0 & 0.011 & $<0.008$ & 29 Apr. 2003 & $0 \neq$ & $0 \neq$ & NA \\
\hline Atrazine $(n=23)$ & 4 & 0 & 0 & 0 & 0.008 & $<0.007$ & 12 Mar. 2003 & $0.061 \neq$ & $0 \neq$ & 12 Mar. 2003 \\
\hline \multirow[t]{5}{*}{ Deethyl atrazine $(n=23)$} & 4 & 0 & 0 & 0 & E0.005 & $<0.006$ & 12 Mar. 2003 & $0.038 \neq$ & $0 \neq$ & 12 Mar. 2003 \\
\hline & & & & & & \multicolumn{2}{|c|}{ Sum of all herbicides analyzed } & $114 \ddagger$ & 16.9 & 12 Mar. 2003 \\
\hline & & & & & & \multicolumn{2}{|c|}{ Sum of all insecticides analyzed } & $2.44 \ddagger$ & 49.3 & 16 Dec. 2003 \\
\hline & & & & & & \multicolumn{2}{|c|}{ Sum of all fungicides analyzed } & $5.28 \neq$ & 15.9 & 12 Feb. 2003 \\
\hline & & & & & & \multicolumn{2}{|c|}{ Sum of all pesticides analyzed } & $122 \ddagger$ & 82.2 & 12 Mar. 2003 \\
\hline
\end{tabular}

†E, estimated concentration; ESA, ethane sulfonic acid; $n$, number of samples; NA, not applicable because $<50 \%$ detections and laboratory reporting limit is greater than this value; $\mathrm{OA}$, oxygen analog.

‡ Only calculated for middle California site to avoid overlap on same date.

thalin is usually incorporated into the soil, it is less available for volatilization into the atmosphere than surface-applied chemicals like atrazine, acetochlor, and alachlor. Alachlor was detected in $58 \%$ of rain samples but at concentrations that were generally one to two orders of magnitude lower than the locally applied pesticides atrazine and acetochlor. Although intermediate- and broad-scale watershed use of alachlor $(1.5$ and $99 \mathrm{Mg} \mathrm{yr}^{-1}$, respectively) was less than half that of pendimethalin (3.6 and $220 \mathrm{Mg} \mathrm{yr}^{-1}$, respectively), alachlor's detection frequency and median concentration were greater (Table 2B), probably because of the difference in their physi$\mathrm{cal} /$ chemical properties. This means that pendimethalin sorbs more strongly to soil and is less likely to volatilize. It also means that airborne pendimethalin is more likely to partition onto particles. Dimethenamid was not detected in 2003 and was detected only twice in 2004. There is little information available in the literature on the physical and chemical properties or the environmental fate of dimethenamid.

Chlorpyrifos was the only insecticide reportedly used in the local-scale watershed in 2003 $\left(103 \mathrm{~kg} \mathrm{yr}^{-1}\right)$. Overall, chlorpyrifos was detected in $54 \%$ of all samples collected (Table 2B). The number of chlorpyrifos detections in 2004 (when there was no reported use in the local-scale watershed) dropped off significantly, with detections in $21 \%$ of the samples. These data indicate that chlorpyrifos may not be transported as far as atrazine and acetochlor partially because of photochemical degradation. Several other insecticides that had no reported use in the local-scale watershed were detected in rain, including malathion (54\%), diazinon (31\%), carbaryl (25\%), and phosmet (20\%) (Table 2B).

\section{Nebraska}

The primary crops grown in the Nebraska study area were corn, soybeans, and alfalfa. Two herbicides (acetochlor and atrazine) and one insecticide (chlorpyrifos) had reported use at all three study-area scales in 2003 and 2004. Metolachlor, atrazine, and acetochlor were the most heavily used herbicides in the broad-scale watershed (473, 462, and $204 \mathrm{Mg} \mathrm{yr}^{-1}$, respectively). Compared with 2003, the amounts of atrazine and acetochlor used in the local-scale watershed were greater in 2004 (15 kg compared with $12 \mathrm{~kg}$ and $38 \mathrm{~kg}$ compared with $31 \mathrm{~kg}$, respectively). This usage was reflected in the detection frequency and concentrations of these herbicides in rain (Fig. 3). The acetochlor concentrations in April and May 2004 were the highest recorded at any of the four sites in this

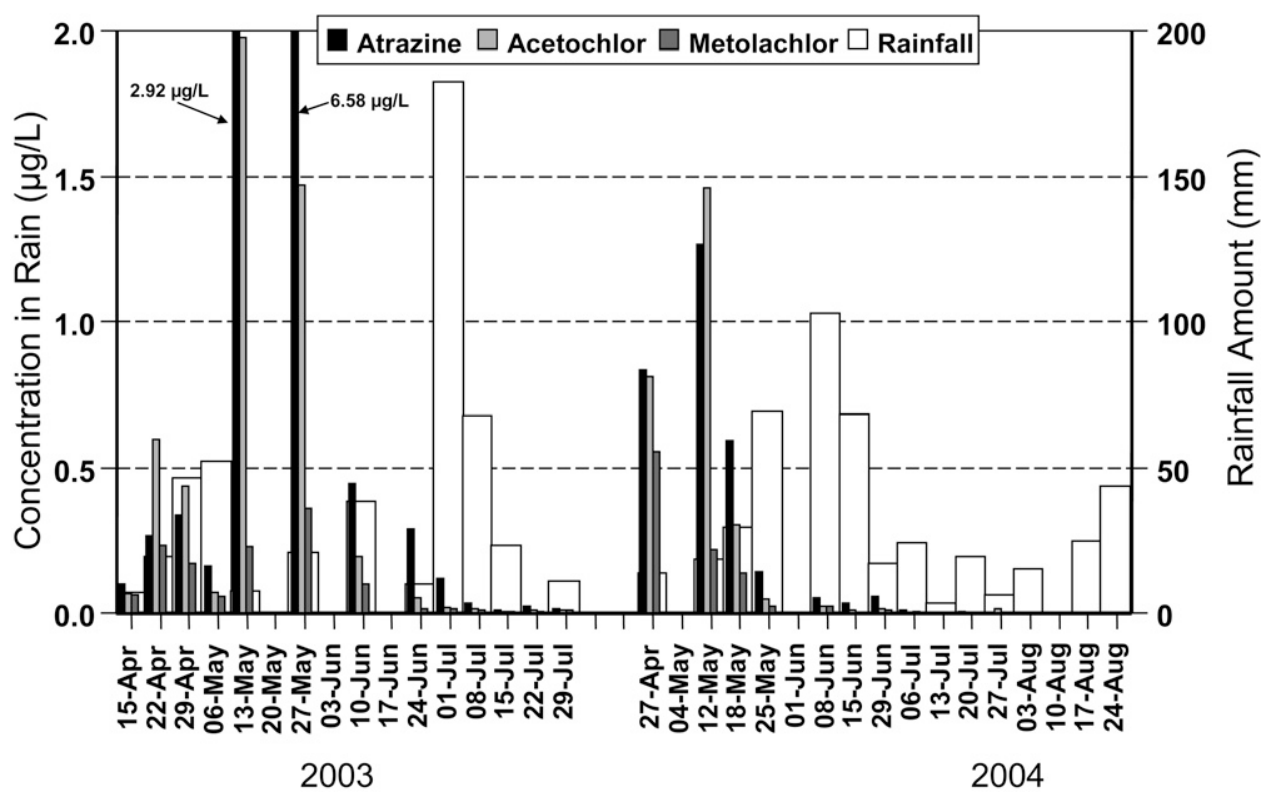

Fig. 2. Atrazine, acetochlor, and metolachlor concentrations in weekly composite rain samples, plus weekly rainfall amounts, at the rain sampling site in Indiana during the growing season in 2003 and 2004. 


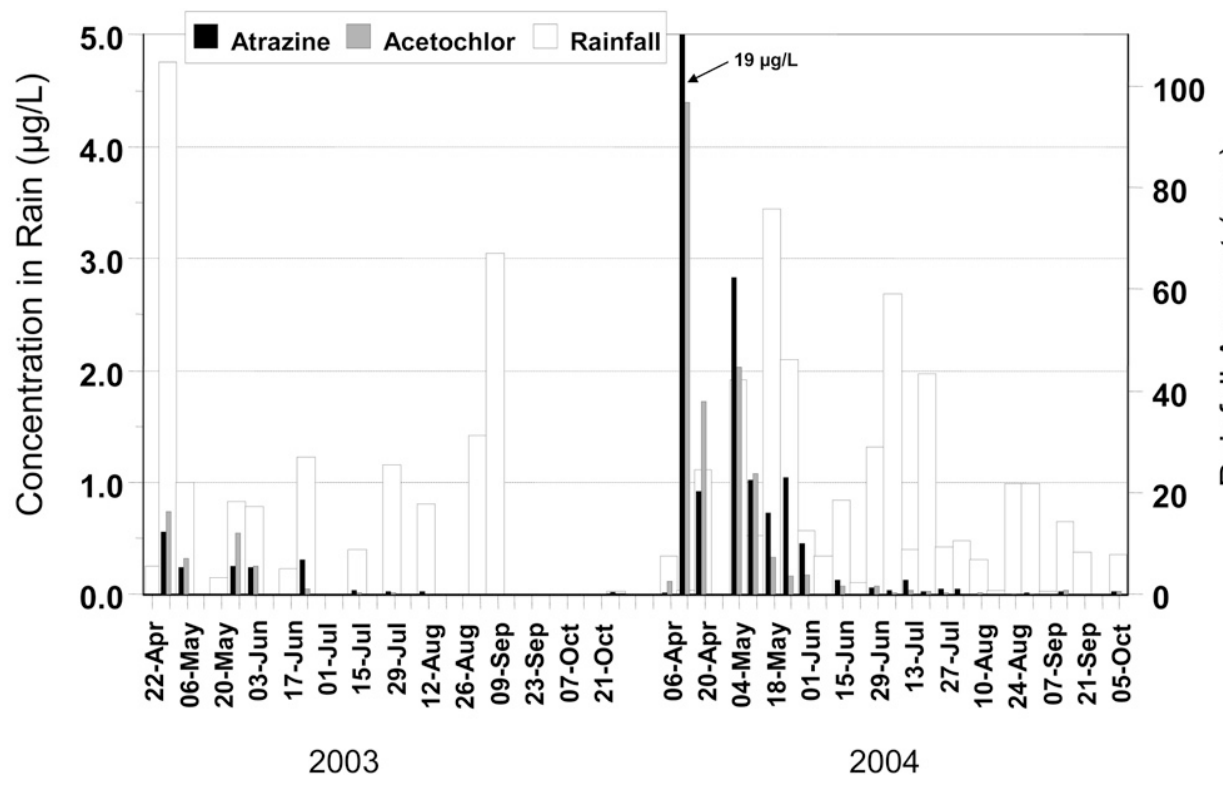

Fig. 3. Atrazine and acetochlor concentrations in weekly composite rain samples, plus weekly rainfall amounts, at the rain sampling site in Nebraska during the growing season in 2003 and 2004.
The herbicides dacthal, metolachlor, and alachlor were frequently detected $(>50 \%)$ but had no reported use in the localscale watershed. Dacthal had no reported use at any watershed scale, and detected concentrations were low (Fig. 4). Even though the intermediate-scale use for metolachlor $(37 \mathrm{Mg}$ $\mathrm{yr}^{-1}$ ) was nearly five times higher than alachlor $\left(7.8 \mathrm{Mg} \mathrm{yr}^{-1}\right)$, the overall detection frequencies for metolachlor and alachlor were similar. The 2004 concentrations and detection frequencies were significantly greater than in 2003 for both herbicides (Fig. 5). Less frequent and generally smaller rainfall events during and after the application period in 2003 were probable factors.

Chlorpyrifos was the most study (maximum of $4.39 \mu \mathrm{g} \mathrm{L}^{-1}$ ) (Table 2C). The highest concentrations of atrazine and acetochlor were observed in a small rain event that occurred shortly after herbicide application to nearby fields.

No trifluralin was reportedly used in the local-scale watershed in 2004, yet the detection frequencies and concentrations were generally similar to 2003, when it was used (Fig. 4). The only exceptions occurred on 27 May 2003 and 3 June 2003 when the trifluralin concentrations were the highest recorded at any of the four sites in this study $\left(0.15\right.$ and $0.060 \mu \mathrm{g} \mathrm{L} \mathrm{L}^{-1}$, respectively). As with atrazine and acetochlor, this sample corresponded to the timing of application in the local-scale watershed in 2003.

frequently detected insecticide at the Nebraska site (74\%) (Table 2C) and was the only insecticide applied in the local-scale watershed. In contrast to the herbicides, the highest concentration was detected in August, late in the growing season. The insecticides methyl parathion, carbaryl, and malathion had reported use in the intermediate- and broad-scale watersheds, but their detection frequencies and concentrations in rain were low (Table 2C). Terbufos was not detected in any rain sample, yet it was the most widely used organophosphorus insecticide in the intermediate- and broad-scale watersheds (4.5 and $57 \mathrm{Mg} \mathrm{yr}^{-1}$, respectively). Terbufos is known to degrade quickly in soil

$\left(\mathrm{t}_{1 / 2}=5 \mathrm{~d}\right)$, which may explain its

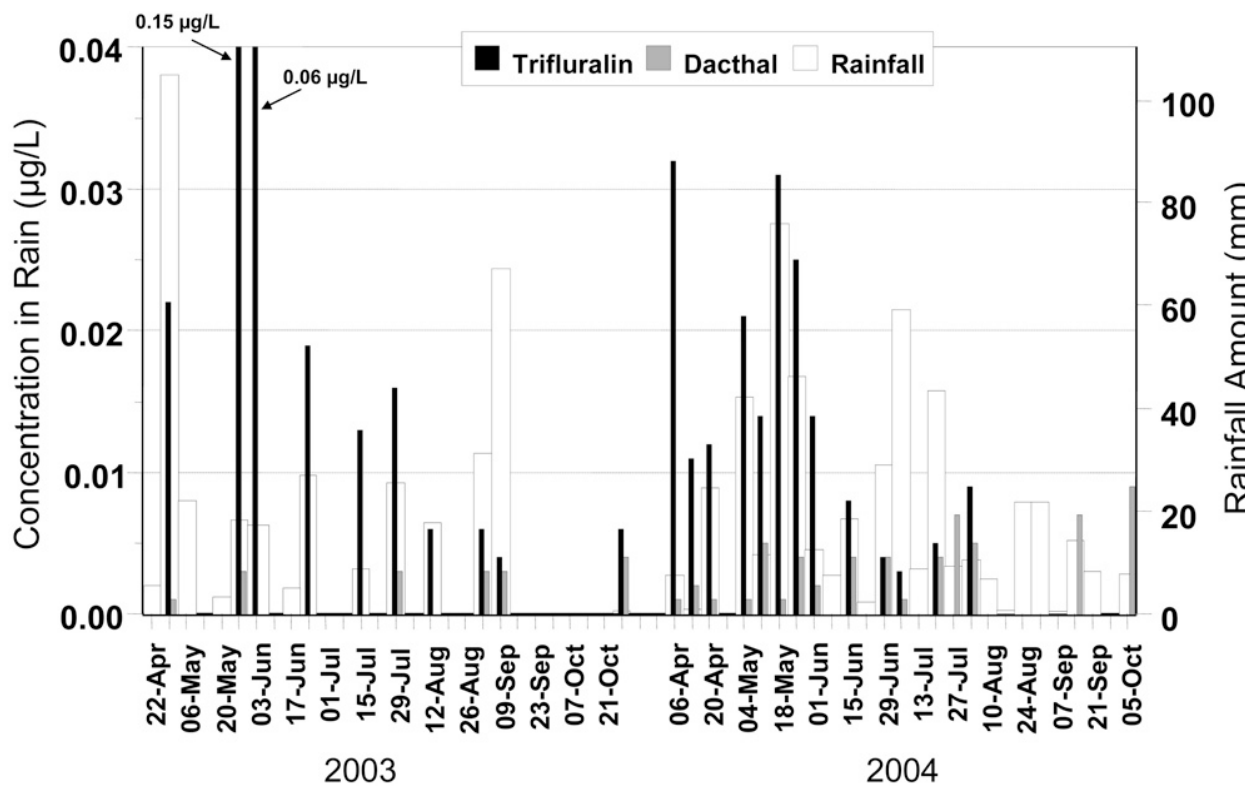

Fig. 4. Trifluralin and dacthal concentrations in weekly composite rain samples, plus weekly rainfall amounts, at the rain sampling site in Nebraska during the growing season in 2003 and 2004. absence in the rain.

\section{California}

The California site contrasted with the other three study sites in that a wide variety of crops were grown (grapes, almonds, corn). Additionally, the growing season and associated pesticide use were reported throughout the year. As is typical, December through March was the rainy season, and rainfall rarely occurred during the summer months. A third difference was that three sites (referred to as upper, middle, and lower, corresponding to their position in the watershed) were sampled instead of one, and the rain samples were event samples rather than weekly composites. For purposes of discussion in this paper, 
samples from 1 Feb. 2003 through 30 Apr. 2003 are considered to represent the 2003 rainy season (samplers were not operating until February), and samples from 1 Dec. 2003 through 30 Apr. 2004 are considered to represent the 2004 rainy season. Insecticides were used more frequently than herbicides in this study area, although both were used extensively. Nine pesticide parent compounds were detected in more than $50 \%$ of the samples: four herbicides, three insecticides, and two fungicides (Table 2D).

Simazine had the highest reported use of the herbicides at all watershed scales in 2003 and 2004. This usage was also reflected in simazine's detection frequency (100\%) and the maximum $\left(15.6 \mu \mathrm{g} \mathrm{L}^{-1}\right)$ and median $\left(0.040 \mu \mathrm{g} \mathrm{L}^{-1}\right)$ concentrations. Metolachlor and trifluralin had similar amounts applied in 2003 in the local-scale watershed, but trifluralin had the higher use at the intermediate- and broadscale watersheds. Trifluralin was detected in $39 \%$ of the samples with a median concentration of $<0.009 \mu \mathrm{g} \mathrm{L}^{-1}$, which was much lower than the other highly used herbicides. Trifluralin is relatively volatile (vapor pressure, $0.0147 \mathrm{~Pa}$ at $25^{\circ} \mathrm{C}$ ), is usually incorporated into the soil at the time of application, and is photoreactive (Woodrow et al., 1978; Mongar and Miller, 1988; Soderquist et al., 1975; Woodrow et al., 1983), which may account for its low detection frequency and concentrations in rain.

Pendimethalin and dacthal had no reported use in the local-scale watershed, but both were used in the intermediate- and broad-scale areas. Pendimethalin had the second-highest use of all herbicides in the broad-scale area $\left(30 \mathrm{Mg} \mathrm{yr}^{-1}\right)$ and was detected frequently (78\%), with a median concentration of $0.021 \mu \mathrm{g} \mathrm{L^{-1 }}$. Dacthal use was considerably lower $\left(4.6 \mathrm{Mg} \mathrm{yr}^{-1}\right)$ but was detected in every rain sample, with a median concentration of $0.009 \mu \mathrm{g} \mathrm{L}^{-1}$.

Of the herbicides commonly used on corn and soybeans and detected at the other three study sites (acetochlor, alachlor, atrazine, and metolachlor), none were reportedly used in the local-scale watershed, and there was minimal or no reported use in the intermediate- and broad-scale watersheds. Atrazine was detected in only one sample, and alachlor and acetochlor were detected in none of the samples. Metolachlor was detected in $61 \%$ of the rain samples collected from the California site but at low concentrations (only $17 \%$ of samples $>0.01 \mu \mathrm{g} \mathrm{L}^{-1}$ ).

The insecticides diazinon, chlorpyrifos, and carbaryl were detected in more than $70 \%$ of the samples; diazinon was present in all of them. All three of these insecticides are typically applied on various crops throughout the year. Major applications of diazinon and chlorpyrifos occurred in January and February for over-wintering insect control in dormant orchards. Chlorpyrifos and carbaryl typically are applied to other crops during this time.
Chlorpyrifos was the most-applied insecticide in the local-, intermediate-, and broad-scale study areas. Diazinon was not used in the local-scale watershed, and its total use in the broad-scale area was about one third that of chlorpyrifos, yet concentrations of diazinon in rain were almost always greater. Diazinon and chlorpyrifos have moderate vapor pressures $\left(3.33 \times 10^{-4}\right.$ and $2.27 \times$ $10^{-3} \mathrm{~Pa}$ at $25^{\circ} \mathrm{C}$, respectively [Capel et al., 2008]), and both are estimated to have short half-lives in air (4.1 and $6.3 \mathrm{~h}$, respectively [Howard et al., 1991]). Diazinon typically has a longer soil half-life than does chlorpyrifos (30 compared with $100 \mathrm{~d}$ ), which would make it available to move into the atmosphere over a longer period of time. Carbaryl is used as a dormant orchard insecticide but is also used on rice, fruit trees, vegetables, grapes, and in nonagricultural areas (California Department of Pesticide Regulation, 2004). The vapor pressure of carbaryl is similar to diazinon $\left(1.60 \times 10^{-4} \mathrm{~Pa}\right.$ at $\left.25^{\circ} \mathrm{C}\right)$, but carbaryl has a shorter typical soil half-life (10 d). Carbaryl undergoes photochemical degradation at a much slower rate than do the other two insecticides (Brahmia and Richard, 2003). Carbaryl was observed in every air sample over a growing season in an agricultural area (grapes, fruit trees, and corn) in Washington (Ohrt et al., 2006).

The fungicides iprodione and myclobutanil were used in California to a much greater extent than in the other study areas (Capel et al., 2008). Only iprodione had any reported use in the local-scale watershed and was detected in $83 \%$ of the samples (Table 2D). The highest concentrations occurred during February of both years (Fig. 6), with an estimated median concentration of $0.074 \mu \mathrm{g} \mathrm{L}^{-1}$. Myclobutanil, which was not used in the local-scale watershed, was detected in $74 \%$ of the rain samples but only in February through April because it is used only after the almond trees bud. The median concentration of myclobutanil was $0.014 \mu \mathrm{g} \mathrm{L}^{-1}$. The physicochemical properties of both fungicides are similar (Capel et al., 2008). Iprodione was applied in greater quantities than myclobutanil at all watershed scales. 

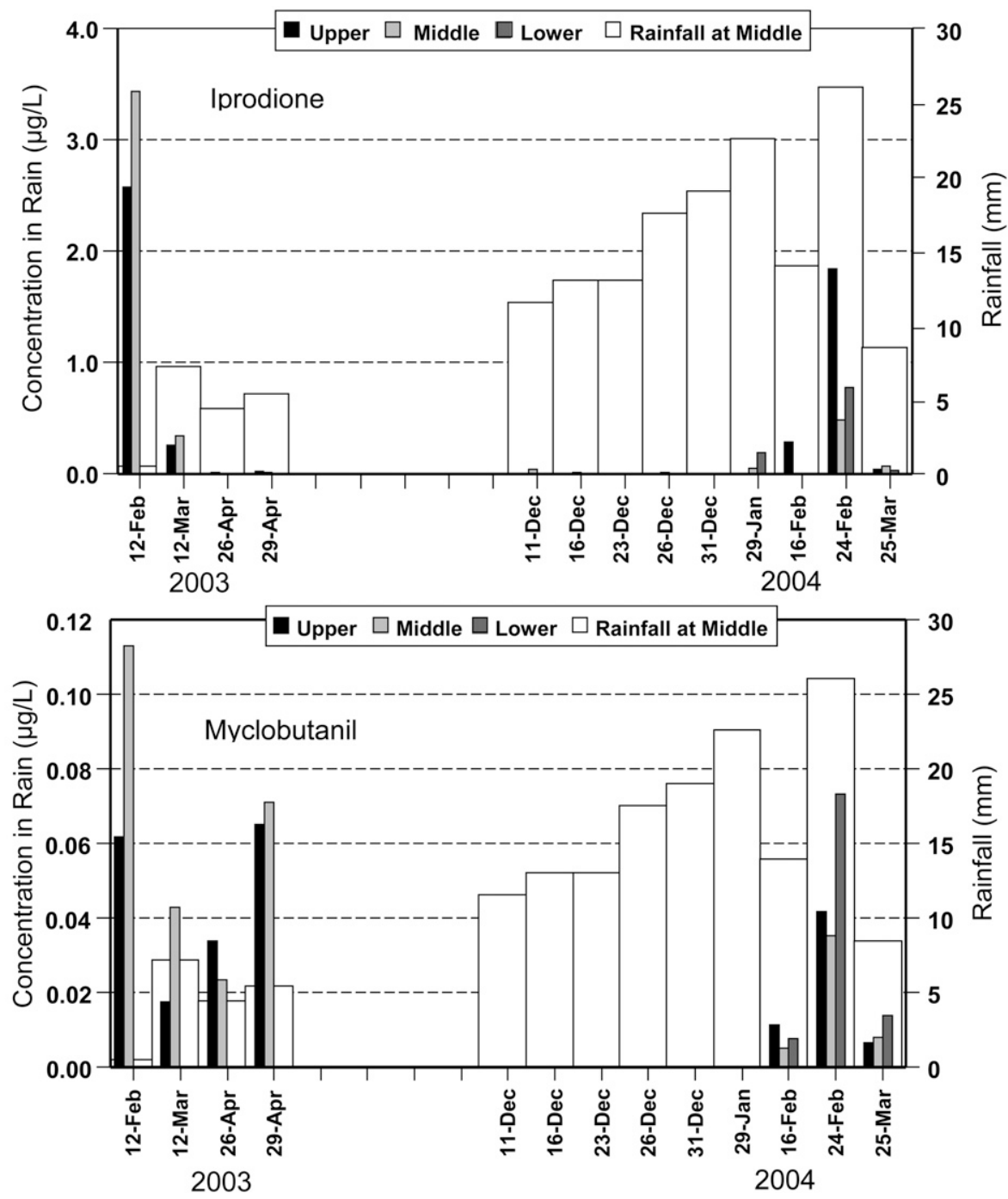

Fig. 6. Iprodione and myclobutanil concentrations in rain samples collected from the upper, middle, and lower sampling sites in California during 2003 and 2004.

\section{Pesticide Degradates}

Pesticides released into the environment are acted on by a number of natural processes that can transform or degrade them into other compounds. In the atmosphere, these processes include reactions with sunlight (photolysis), with other airborne contaminants like ozone and hydroxyl radicals (oxidation), and hydrolysis. The resulting transformation products (commonly referred to as degradates) generally are more water soluble than the parent and usually less toxic. Degradates of some pesticides, notably organophosphate insecticides like chlorpyrifos, are much more toxic than the parent compound (Wu and Laird, 2003).

The samples collected in this study were analyzed for $40 \mathrm{deg}$ radates. The most frequently detected were deethylatrazine (DEA) (from atrazine), chlorpyrifos oxygen analog $(\mathrm{OA})$, diazinon $\mathrm{OA}$, malathion OA, and 1-napthol (primarily from carbaryl). Three distinct patterns emerged from analysis of the degradate data. First, DEA was detected in about two thirds of the samples collected at the Midwestern and Eastern study sites (Maryland, 59\%; Indiana, $73 \%$; and Nebraska, $75 \%$ ), but DEA was detected only once in California. The occurrence of DEA in rain samples mirrors the use of atrazine at the study sites. Second, detections of the OAs of chlorpyrifos, diazinon, and malathion occurred primarily in California. Malathion OA also was detected in samples from Indiana (36\%) and Nebraska (18\%), but chlorpyrifos OA was rarely detected at the Midwestern and Eastern sites, and diazinon OA was not detected. The detection frequencies and concentrations of the detected OA degradates usually reflected the amount of the parent compound used. Third, degradates of the acetanilide herbicides (acetochlor, alachlor, and metolachlor) were rarely detected in rain. The ethane sulfonic acid, oxanilic acid, and the sulfynil acetic acid degradates of the acetanilide herbicides are frequently found in surface and ground waters at relatively high concentrations (Scribner et al., 2004). The formation of these degradates is a microbially mediated process (Barbash, 2003) that would not be expected to occur in the atmosphere or falling rain droplets. Because these compounds are organic acids, they do not undergo volatilization in their ionic forms from soil or surface water.

The degradate fraction is the molar ratio of a degradate to its parent compound plus all degradates. The degradate fraction is calculated using Eq. [1] and molar units for all concentrations. This equation assumes a stoichiometric degradation and makes no assumptions about how the parent compound degrades.

$$
\text { Degradate(i) Fraction }=\frac{\text { Degradate }(\mathrm{i})}{\sum[\text { Degradates }]+[\text { Parent }]}
$$

The degradate fraction can be used to investigate the behavior of a degradate relative to its parent. In the situation where data are available for a parent pesticide and only one of its degradates, a degradate fraction equal to unity means that only the degradate is present and that no parent pesticide was detected (assuming a unique parent pesticide). Conversely, a degradate fraction of zero means that only the parent compound was present and that no degradate(s) was detected. For the situation in which multiple degradates are analyzed, a degradate fraction equal to one indicates that only the degradate of interest is present. If multiple degradates are analyzed and the degradate fraction equals zero, then there is no degradate(i). There may be other degradates or the parent pesticide present.

Figure 7 shows the degradate fraction for DEA in the Maryland, Indiana, and Nebraska rain samples and the cumulative rain- 
fall amounts in 2003 and 2004. Deethylatrazine was detected in only one California rain sample and is not included in this graph. Deisopropyl atrazine and other atrazine degradates were not analyzed in this study and are not included in the DEA fraction calculation. The DEA fraction tended to gradually increase after the herbicide applications in the spring and peaked near mid-summer, then decreased rapidly or was no longer detected. The peak DEA fraction for Maryland, Indiana, and Nebraska samples in 2003 was measured on 8 July 2003 (degradate fraction, 0.50; last detection of atrazine and DEA), 15 July 2003 (0.57), and 29 July 2003 (0.71), respectively. In 2004, peak DEA fractions for Maryland, Indiana, and Nebraska samples were measured on 15 June 2004 (0.55), 8 June 2004 (0.36), and 20 July 2004 (0.68), respectively. Compared with 2003, the peak DEA fraction occurred sooner at about the same magnitude in 2004 in Maryland, occurred sooner with a lower peak value in 2004 in Indiana, and occurred at a similar time with similar magnitude in 2004 in Nebraska. The interannual differences may reflect different cropping patterns, precipitation, timing of application, or other climate variables. The largest DEA fraction was observed in Nebraska during both years. The gradual upward trend in the DEA fraction was not readily observable in a plot of DEA concentration values (not shown) in which concentrations began to decline shortly after the application period in most instances.

The degradates chlorpyrifos OA, malathion OA, diazinon $\mathrm{OA}$, and 1-naphthol were detected mostly during their periods of use in each of the study areas. The most distinguishable trends in degradate fraction values for these compounds were at the Indiana and California sites (Fig. 8). For the California sites, the upper, middle, and lower sampling sites are included on the same plot. The degradate fraction values in Indiana for chlorpyrifos OA and malathion OA returned to nondetectable levels within 6 wk of initial detection (Fig. 8A, 8C, 8D). In California, considering the low concentrations found in these samples, the degradate fractions of chlorpyrifos OA, diazinon $\mathrm{OA}$, and 1-napthol seem to be relatively constant to increasing throughout the period of detection (Fig. $8 \mathrm{~B}, 8 \mathrm{E}, 8 \mathrm{~F}$ ), which is reflective of their relatively short persistence in the atmosphere and timings of application, which may be spaced throughout the period of detection.

Deethylatrazine tends to persist in rain for a longer period of time after application of the parent compound than do the organophosphate OA degradates detected during this study. Additionally, the DEA fraction in rain shows an increasing trend throughout the summer months after application, whereas the degradate fractions of the insecticide degradates show no consistent trend during or after the period of application in the area.

\section{Pesticide Deposition}

The total weekly (or rain event in California) wet deposition of each pesticide analyzed in the study was calculated using the measured concentrations and total precipitation amounts (Table 2). Total wet deposition of pesticides in California was calculated using data from the middle site only. The total wet deposition at the Maryland, Indiana, and Nebraska sites was dominated by herbicides, which accounted for at least 91 to $98 \%$ of the total pesticide mass deposited (Fig. 9). This was not unexpected because herbicide use greatly exceeded insecticide use at these sites where corn and soybeans were the predominant crops. The largest contributors to the depositional mass of herbicides at these three sites were atrazine, acetochlor, and metolachlor (plus pendimethalin in Maryland). Carbaryl, malathion, malathion OA, and chlorpyrifos were the insecticide compounds composing the largest percentage of the insecticide mass deposited by the rain. At the middle site in California, 21\% of the total pesticide wet deposition consisted of herbicides in 2004, compared with $94 \%$ of total pesticide deposition in the partially monitored season of 2003 (most of this herbicide deposition was from one event and was probably caused by drift from a local application). In California in 2004, the herbicides simazine (11\%) and pendimethalin (4\%) accounted for about three fourths of the herbicide component of the total deposited mass of pesticides in rain; however, insecticides accounted for $60 \%$ of the total wet deposition at the California site in 2004. Insecticides that contributed most of the total mass of pesticides in all wet deposition in California included diazinon, methidathion, and chlorpyrifos. Additionally, the fungicide iprodione ranked third in mean yearly wet deposition.

Total wet deposition of the various pesticides in Maryland was similar in 2003 and 2004, reflecting similar application rates at the local scale. By contrast, total wet mass deposition of various pesticides in Indiana and Nebraska differed between 2003 and 2004. This was partly the result of variable year-to-year glypho- 

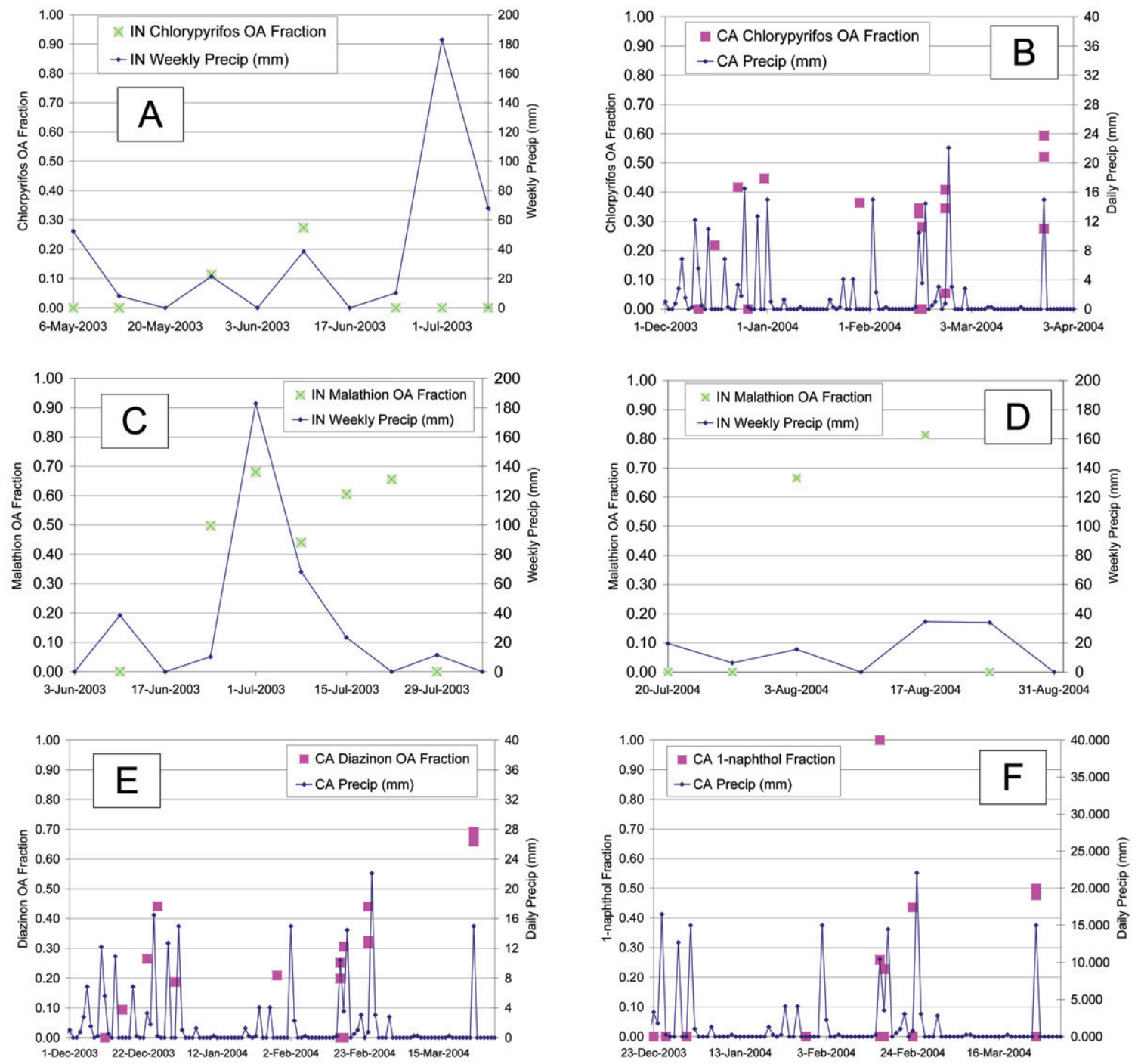

Fig. 8. Degradate fractions in rain samples and rainfall amounts during selected time periods for (a) chlorpyrifos oxygen analog (OA) at the Indiana site in 2003, (b) chlorpyrifos OA at the California sites in late 2003 and early 2004, (c) malathion OA at the Indiana site in 2003,

(d) malathion OA at the Indiana site in 2004, (e) diazinon OA at the California sites in late 2003 and early 2004, and (f) 1-naphthol at the California sites in December 2003.

sate application (which may have taken the place of the acetanilide or triazine herbicides for weed control and was not measured in the rain samples from this study) during the normal crop

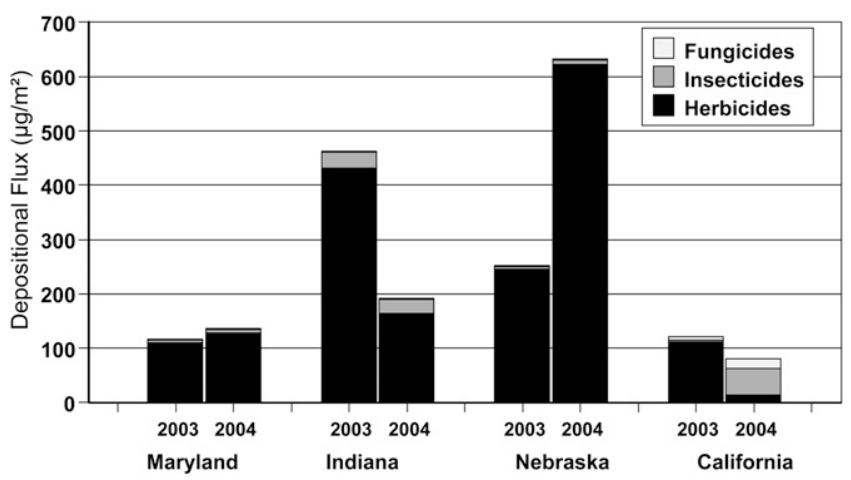

Fig. 9. Total seasonal mass deposition of herbicides, insecticides, and fungicides in rain at the Maryland, Indiana, Nebraska, and California sites in 2003 and 2004 rotation of soybeans and corn. Timing and frequency of rainfall at each of the sites in relation to pesticide application dates also may have played a large role in the year-to-year variability of total wet deposition of pesticides. Year-to-year total mass deposition comparisons in California between the 2003 rainy season and the 2004 rainy season cannot be made because rain samples were collected during only part of the 2003 rainy season.

\section{Local versus Long-Range Sources}

The data from this study show that proximity to application areas strongly influences the occurrence of pesticides in rainfall. For example, in Nebraska the local-scale use of trifluralin in 2003 resulted in high detection rates and concentrations in rain during the application season. Majewski et al. (1998) found similar results for trifluralin in airborne pesticide residues along the Mississippi River. The absence of local-scale pesticide use at the Indiana site in 2004 (for pesticides that were analyzed during this study) was likely largely responsible for a $70 \%$ decrease in atrazine wet 
deposition and 63\% decrease in DEA wet deposition in 2004 compared with 2003. In fact, most pesticides having a detection frequency $>10 \%$ were detected less frequently in 2004 than in 2003 at the Indiana site, where only prometon, malathion, and malathion OA were detected more frequently in 2004.

Although local pesticide use is a major determinant of the types and mass of pesticides present in wet deposition, regionaland long-range atmospheric transport may be responsible for a portion of the mass loading of some pesticides in some watersheds. Insecticides and herbicides were detected in rain samples in watersheds with no reported local-scale use of those compounds. Insecticides detected in samples from areas with no reported localscale use included malathion, carbaryl, diazinon, and methidathion, indicating long-range atmospheric transport from use in the intermediate- or broad-scale watershed. Malathion and malathion OA were detected in Nebraska (sporadic, low-level detections) and in Indiana (consistent, lowlevel detections during July and August of both years) despite the absence of reported agricultural use of the insecticide at any scale in Nebraska and only broad-scale use reported in Indiana. Assuming accurate pesticide usage reports, the malathion and malathion OA detections were likely a result of long-range atmospheric transport of the insecticide into the study area. Carbaryl also had the third highest annual mean wet deposition of all insecticides in Nebraska (after chlorpyrifos and azinphos-methyl, both of which had greater intermediate- and broad-scale use), even though there was no reported local use of carbaryl.

In California, diazinon was detected in all rain samples collected, even though there was no reported local-scale use but substantial use at the intermediate scale. This result indicates that intraregional atmospheric transport throughout the rainy season was the source of diazinon in rain. Diazinon was detected in the stream water of Mustang Creek, which drains the local-scale area. Because there was no use in the watershed, the only measured or reported source of the pesticide in the watershed was from the rainfall (Domagalski et al., 2008). As evidence for intraregional transport, the highest concentrations in rain for carbaryl and methidathion at the California sampling sites occurred on 16 Dec. 2003 (Table 2D), well before the dormant orchard spraying normally begins in the local-scale area. These insecticides were used on other crops at the intermediate and broad scales and likely were transported atmospherically into the local-scale area.

Several herbicides were found in rain falling in areas with no reported local use of the compounds, including pendimethalin, acetochlor, and dacthal. Pendimethalin was found in multiple samples at all four study areas, even though no local use was reported for any of the sites. There was substantial use of pendimethalin at the intermediate scale of all four study areas; in mass, applied pendimethalin ranked fourth nationally among the pesticides analyzed in this study (Table 1). This result indicates that the mid- to long-range transport of pendimethalin is important. Similarly, acetochlor was detected in rain at the Maryland site where there was no reported local-scale use. There was, however, substantial use in the intermediate- and broad-scale watersheds. When compared with acetochlor concentrations in rain in the other corn/soybean areas in Indiana and Nebraska, however, the median acetochlor concentrations measured in Maryland were at least five to seven times lower, consistent with a longer transport distance from the source to the sampling site. Dacthal was detected at low levels at every site and in $70 \%$ of all samples. The low concentrations observed at all the study sites throughout each sampling season indicate that dacthal is well mixed in the atmosphere and likely undergoing long-range transport. Other studies have shown dacthal to be environmentally stable, widely detected in the atmosphere (Foreman et al., 2000; Majewski et al., 2000), and prone to long-range transport (Rice and Chernyak, 1997).

\section{Timing of Pesticide Deposition}

Figure 10 illustrates that a large percentage of the total wet deposition at these sites is often related to a few weekly samples or storm events. Generally, the substantial deposition is associated with large storms that occur shortly after pesticide applications in the local area. Most of the instances of this phenomenon at the four sites analyzed during this study involved herbicides (simazine, atrazine, acetochlor, metolachlor, and alachlor), although one storm in California in December 2003 deposited a large mass of two insecticides (diazinon and methidathion). During the week of 20 Apr. 2004, in Maryland, metolachlor and atrazine deposition $\left(35 \mathrm{\mu g} \mathrm{m}^{-2}\right)$ was $26 \%$ of the year's total wet deposition of pesticide at that site. In Indiana, wet deposition of atrazine and acetochlor during the week of 27 May 2003 accounted for 37\% 
Table 3. Total mass of pesticides deposited by rain onto study areas of various scales given the assumption of uniform distribution of rainfall over the entire area in (A) 2003 and (B) 2004 and annual wet deposition of pesticides relative to total mass of pesticide applied in (C) 2003 and (D) 2004.

\begin{tabular}{|c|c|c|c|c|}
\hline & Maryland & Indiana & Nebraska & California \\
\hline & & & $-\mathrm{kg}$ & \\
\hline \multicolumn{5}{|l|}{ A. Total pesticides deposited, 2003} \\
\hline Total in local-scale area & 3.84 & 3.32 & 0.379 & partial season \\
\hline Total in intermediate-scale area & 84 & 112 & 241 & partial season \\
\hline Total in broad-scale area & 614 & 13,572 & 3841 & partial season \\
\hline \multicolumn{5}{|c|}{ B. Total pesticides deposited, 2004} \\
\hline Total in local-scale area & 4.41 & 1.37 & 0.942 & 3.23 \\
\hline Total in intermediate-scale area & 96.7 & 46.3 & 600 & 154 \\
\hline Total in broad-scale area & 705 & 5603 & 9542 & 2875 \\
\hline \multicolumn{5}{|l|}{ C. Percent of applied, 2003} \\
\hline In local-scale area & 0.06 & 0.94 & 0.53 & partial season \\
\hline In intermediate-scale area & 0.05 & 0.30 & 0.20 & partial season \\
\hline In broad-scale area & 0.21 & 0.53 & 0.24 & partial season \\
\hline \multicolumn{5}{|l|}{ D. Percent of applied, 2004} \\
\hline In local-scale area & 0.06 & NA & 1.73 & 0.09 \\
\hline In intermediate-scale area & 0.06 & 0.12 & 0.49 & 0.24 \\
\hline In broad-scale area & 0.24 & 0.22 & 0.60 & 0.29 \\
\hline
\end{tabular}

$\left(171 \mathrm{~g} \mathrm{~m} \mathrm{~m}^{-2}\right)$ of that year's wet deposition total. In Nebraska, wet deposition of atrazine, acetochlor, metolachlor, and alachlor during the weeks of 29 Apr. 2003, 4 May 2004, and 18 May 2004 contributed 66\% (166 $\left.\mu \mathrm{g} \mathrm{m}^{-2}\right), 37 \%\left(233 \mu \mathrm{g} \mathrm{m}^{-2}\right)$, and $18 \%\left(114 \mathrm{\mu g} \mathrm{m}^{-2}\right)$, respectively, of the year's total wet deposition of all pesticides. From individual storm data in at the middle site in California, simazine accounted for $93 \%\left(113 \mu \mathrm{g} \mathrm{m}^{-2}\right)$ of total pesticide wet deposition between February and April 2003. From a single storm sample collected 16 Dec. 2003, the mass of the insecticides diazinon and methidathion accounted for 31\% (25 $\mu \mathrm{g} \mathrm{m}^{-2}$ ) of the total wet deposition of all pesticides during the rainy season of 2003 to 2004 (November through April).

\section{Relative Contributions}

The contribution of pesticides in rain can be compared with the total pesticide mass applied to a watershed to determine the relative contribution of wet deposition of pesticides to the total input of pesticides in an area. Tables $3 \mathrm{~A}$ and $3 \mathrm{~B}$ show the deposition of pesticides (total mass and as a percent of application) to the studied watersheds with the assumption of uniform distribution of rainfall over the entire basin. Although this may not be an accurate assumption for the larger-scale watersheds, it is an estimate of the magnitude of the mass deposited in these predominantly agricultural areas. Relative to the total mass of pesticides applied, the growing season total wet deposition of pesticides in rainfall at the local scale ranged from 0.06 to $1.73 \%$ (Tables 3C and 3D). At the broad scale, the wet deposition of pesticides relative to the total mass applied was less variable (range, $0.21-0.60 \%$ ). By comparison, the mass deposited by rain relative to the amount applied was less in Maryland than in Nebraska and Indiana in 2003 (California was only a partial year in 2003), whereas in 2004 the ratio for Nebraska was greater than that for Maryland, Indiana, or California.

\section{Acknowledgments}

This research was supported by the U.S. Geological Survey (USGS), National Water-Quality Assessment (NAWQA) Program. We thank the landowners who allowed the researchers access to their agricultural fields. We also thank the numerous USGS employees who assisted with installation, sample collection, and data management for their involvement in the successful completion of the project. Finally, we thank M. Alisa Mast and Hank Johnson for their excellent suggestions during the USGS internal colleague review process. Any use of trade, firm, or product names is for descriptive purposes only and does not imply endorsement by the U.S. Government.

\section{References}

Asman, W.A.H., A. Jørgensen, R. Bossi, K.V. Vejrup, B.B. Mogensen, and M. Glasius. 2005. Wet deposition of pesticides and nitrophenols at two sites in Denmark: Measurements and contributions from regional sources. Chemosphere 59:1023-1031.

Aulagnier, F., and L. Poissant. 2005. Some pesticides occurrence in air and precipitation in Québec, Canada. Environ. Sci. Technol. 39:2960-2967.

Barbash, J.E. 2003. The geochemistry of pesticides. p. 541-577. In H.D. Holland and K.K. Turekian (ed.) Treatise on geochemistry. Pergamon, Oxford, UK.

Bedos, C., P. Cellier, R. Calvet, E. Barriuso, and B. Gabrielle. 2002. Mass transfer of pesticides into the atmosphere by volatilization from soils and plants: Overview. Agronomie (Paris) 22:21-33.

Bidleman, T.F. 1988. Atmospheric processes: Wet and dry deposition of organic compounds are controlled by their vapor-particle partitioning. Environ. Sci. Technol. 22:361-367.

Bidleman, T.F. 1999. Atmospheric transport and air-surface exchange of pesticides. Water Air Soil Pollut. 115:115-166.

Bigelow, D.S., S.R. Dossett, and V.C. Bowersox. 2001. Instruction manualNADP/NTN site operation. National Atmospheric Deposition Program (NADP) manual 2000-1. Illinois State Water Survey, Champaign, IL.

Brahmia, O., and C. Richard. 2003. Phototransformation of carbaryl in aqueous solution: Laser-flash photolysis and steady-state studies. J. Photochem. Photobiol. A 156:9-14.

California Department of Pesticide Regulation. 2004. Pesticide use data for 2003 [digital data]. California Dep. of Pesticide Regulation, Sacramento, CA.

Capel, P.D., and S.J. Larson. 1995. A chemodynamic approach for estimating losses of target organic chemicals from water during sample holding time. Chemosphere 30:1098-1107.

Capel, P.D., K.A. McCarthy, and J.E. Barbash. 2008. National holistic watershed-scale approach for studying agricultural chemicals. J. Environ. Qual. 37:983-993.

Carlson, D.L., I. Basu, and R.A. Hites. 2004. Annual variations of pesticide concentrations in Great Lakes precipitation. Environ. Sci. Technol. 38:5290-5296.

Charizopoulos, E., and E. Papadopoulou-Mourkidou. 1999. Occurrence of pesticides in rain of the Axios River basin, Greece. Environ. Sci. Technol. 33:2363-2368.

De Rossi, C., R. Bierl, and J. Riefstahl. 2003. Organic pollutants in precipitation: Monitoring of pesticides and polycyclic aromatic hydrocarbons in the region of Trier (Germany). Phys. Chem. Earth 28:307-314.

Domagalski, J.L., S. Ator, R. Coupe, K. McCarthy, D. Lampe, M. Sandstrom, and N. Baker. 2008. Comparative study of transport processes of agricultural chemicals to streams. J. Environ. Qual. 37:1158-1169.

Foreman, W.T., M.S. Majewski, D.A. Goolsby, F.W. Wiebe, and R.H. Coupe. 2000. Pesticides in the atmosphere of the Mississippi River Valley: Part II. Air. Sci. Total Environ. 248:213-226.

Glotfelty, D.E., G.H. Williams, H.P. Freeman, and M.M. Leech. 1990. Regional atmospheric transport and deposition of pesticides in Maryland. p. 199-222. In D. Kurtz (ed.) Long range transport of pesticides. Lewis Publ., Chelsea, MI.

Goel, A., L.L. McConnell, and A. Torrents. 2005. Wet deposition of current use pesticides at a rural location on the Delmarva Peninsula: Impact of rainfall patterns and agricultural activity. J. Agric. Food Chem. 53:7915-7924. 
Howard, P.H., E.M. Michalenko, W.F. Jarvis, D.K. Basu, G.W. Sage, W.M. Meylan, J.A. Beauman, and D.A. Gray. 1991. Handbook of environmental fate and exposure data for organic chemicals. Volume III, Pesticides. Lewis Publ., Chelsea, MI.

Majewski, M.S., and P.D. Capel. 1995. Pesticides in the atmosphere: Distribution, trends, and governing factors. Ann Arbor Press, Chelsea, MI

Majewski, M.S., W.T. Foreman, and D.A. Goolsby. 2000. Pesticides in the atmosphere of the Mississippi River Valley. Part I. Rain. Sci. Total Environ. 248:201-212.

Majewski, M.S., W.T. Foreman, D.A. Goolsby, and N. Nakagaki. 1998. Airborne pesticide residues along the Mississippi River. Environ. Sci. Technol. 32:3689-3698.

Martin, J.D., C.G. Crawford, and S.J. Larson. 2002. Pesticides in streams: Summary statistics; preliminary results from cycle I of the National Water-Quality Assessment Program (NAWQA), 1992-2001. USGS online report. Available at http://ca.water.usgs.gov/pnsp/pestsw/PestSW_2001_Text.html (verified 26 June 2006).

Miller, S.M., C.W. Sweet, J.V. Depinto, and K.C. Hornbuckle. 2000. Atrazine and nutrients in precipitation: Results from the Lake Michigan mass balance study. Environ. Sci. Technol. 34:55-61.

Mongar, K., and G.C. Miller. 1988. Vapor phase photolysis of trifluralin in an outdoor chamber. Chemosphere 17:2183-2188.

Ohrt, A.W., P.D. Capel, and R.W. Black. 2006. Concentrations of current-use agricultural pesticides in the air, Yakima County, Washington. p. 174-178. In V.P. Aneja (ed.) Proc. Workshop on Agricultural Air Quality: State of the Science, 5-8 June 2006. North Carolina State Univ., Raleigh, NC

Park, J.-S., T.L. Wade, and S.T. Sweet. 2001. Atmospheric deposition of organochlorine contaminants to Galveston Bay, Texas. Atmos. Environ. 35:3315-3324.

Park, J.-S., T.L. Wade, and S.T. Sweet. 2002. Atmospheric deposition of $\mathrm{PAHs}$, PCBs, and organochlorine pesticides to Corpus Christi Bay, Texas. Atmos. Environ. 36:1707-1720.
Polkowska, Z., A. Kot, M. Wiergowski, L. Wolka, K. Wolowska, and J. Namieśnik. 2000. Organic pollutants in precipitation: Determination of pesticides and polycyclic aromatic hydrocarbons in Gdańsk, Poland. Atmos. Environ. 34:1233-1245.

Rice, C.P., and S.M. Chernyak. 1997. Marine Arctic fog: An accumulator of currently used pesticide. Chemosphere 35:867-878.

Scribner, E.A., J.E. Dietze, and E.M. Thurman. 2004. Acetamide herbicides and their degradation products in ground water and surface water of the United States, 1998-2003. USGS Data Series 88. USGS, Washington, DC.

Soderquist, C.J., D.G. Crosby, K.W. Moilanen, J.N. Seiber, and J.E. Woodrow. 1975. Occurrence of trifluralin and its photoproducts in air. J. Agric. Food Chem. 23:304-309.

Thelin, G.P., and L.P. Gianessi. 2000. Method for estimating pesticide use for county areas of the conterminous United States: USGS Open-File Report 00-250. USGS, Washington, DC.

Thurman, E.M., and A.E. Cromwell. 2000. Atmospheric transport, deposition, and fate of triazine herbicides and their metabolites in pristine areas at Isle Royale National Park. Environ. Sci. Technol. 34:3079-3085.

Van Dijk, H.F.G., and R. Guicherit. 1999. Atmospheric dispersion of current-use pesticides: A review of the evidence from monitoring studies. Water Air Soil Pollut. 115:21-70.

Waite, D.T., P. Bailey, J.F. Sproull, D.V. Quiring, D.F. Chau, J. Bailey, and A.J. Cessna. 2005. Atmospheric concentrations and dry and wet deposits of some herbicides currently used on the Canadian Prairies. Chemosphere 58:693-703.

Woodrow, J.E., D.G. Crosby, T. Mast, K.W. Moilanen, and J.N. Seiber. 1978. Rates of transformation of trifluralin and parathion vapors in air. J. Agric. Food Chem. 26:1312-1316.

Woodrow, J.E., J.N. Seiber, and D.G. Crosby. 1983. Vapor-phase photochemistry of pesticides. Residue Rev. 85:111-125.

Wu, J., and D.A. Laird. 2003. Abiotic transformation of chlorpyrifos to chlorpyrifos oxon in chlorinated water. Environ. Toxicol. Chem. 22:261-264. 